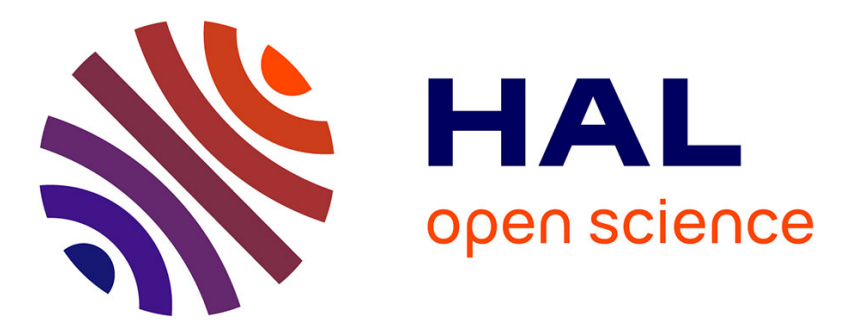

\title{
Vector borne diseases on an urban environment: the effects of heterogeneity and human circulation
}

Abderrahman Iggidr, Jair Koiller, Maria Lucia F. Penna, Gauthier Sallet, Moacyr Alvim Silva Silva, Max O. Souza

\section{To cite this version:}

Abderrahman Iggidr, Jair Koiller, Maria Lucia F. Penna, Gauthier Sallet, Moacyr Alvim Silva Silva, et al. Vector borne diseases on an urban environment: the effects of heterogeneity and human circulation. Ecological Complexity, 2017, 30, pp.76-90. 10.1016/j.ecocom.2016.12.006 . hal-01422432

HAL Id: hal-01422432

https://hal.inria.fr/hal-01422432

Submitted on 25 Dec 2016

HAL is a multi-disciplinary open access archive for the deposit and dissemination of scientific research documents, whether they are published or not. The documents may come from teaching and research institutions in France or abroad, or from public or private research centers.
L'archive ouverte pluridisciplinaire HAL, est destinée au dépôt et à la diffusion de documents scientifiques de niveau recherche, publiés ou non, émanant des établissements d'enseignement et de recherche français ou étrangers, des laboratoires publics ou privés. 


\title{
Vector borne diseases on an urban environment: the effects of heterogeneity and human circulation*
}

\author{
A. Iggidr ${ }^{1}$, J. Koiller ${ }^{2,3}$, M.L.F. Penna ${ }^{4}$, G. Sallet ${ }^{1}$, M.A. Silva ${ }^{5}$, and \\ M.O. Souza ${ }^{\dagger 6}$ \\ ${ }^{1}$ Inria, Université de Lorraine, and CNRS, IECL (UMR 7502), ISGMP Bat. A, \\ Ile du Saulcy, 57045 Metz Cedex 01, France \\ ${ }^{2}$ Instituto Nacional de Metrologia, Qualidade e Tecnologia (INMETRO), Av. \\ Nossa Senhora das Graças 50 , Duque de Caxias - RJ, Brazil, 25250-020 \\ ${ }^{3}$ Instituto Politécnico, Universidade do Estado do Rio de Janeiro, Rua Bonfim \\ 25, Friburgo, RJ, Brazil, 28625-570 \\ ${ }^{4}$ Departamento de Epidemiologia e Bioestatística, Instituto de Saúde da \\ Comunidade,Universidade Federal Fluminense, Rua Marquês de Paraná 303, \\ Niterói, Rio de Janeiro, Brazil, 24030-210 \\ ${ }^{5}$ Escola de Matemática Aplicada, Fundação Getulio Vargas , Praia de Botafogo \\ 190, Rio de Janeiro, RJ, Brazil, 22250-040 \\ ${ }^{6}$ Departamento de Matemática Aplicada, Universidade Federal Fluminense , Rua \\ Mário Santos Braga, s/n, Niterói, Rio de Janeiro, Brazil, 24020-140
}

\begin{abstract}
We study the effect of human circulation and host/vector heterogeneities on the onset of epidemics of arboviruses. From a meta-population dynamics based on the classical BaileyDietz model, we derive a multi-group model under three assumptions: (i) fast host sojourn time-scale; (ii) mosquitoes do not move; (iii) time homogeneity and strong connectivity of human circulation. Within this modelling framework, three different kinds of $R_{0}$ appear: (i) the "true" or "global" $R_{0}$-derived from the corresponding next generation matrix; (ii) the uniform $R_{0}$-obtained if the patches are taken homogeneous; (iii) the local $R_{0}$ s-obtained if the patches are disconnected. We show that there is relevant epidemiological information associated to all of them. In particular, they can be used to understand the effects of changing the circulation on the value of the global $R_{0}$. We also present additional results on the effects on $R_{0}$ of different vector control policies, and a simulation with data from the city of Rio de Janeiro, Brazil.
\end{abstract}

Keywords. urban epidemics; meta-population models; multi-scaling reduction; basic reproductive number; arboviruses

${ }^{*}$ This work was supported by the CNPq Pronex/Dengue network grant \# 550030/2010-7 and by COFECUB/CAPES joint grant 709-2010.

${ }^{\dagger}$ MOS was partially supported by CNPq grants \# 308113/2012-8, \# 486395/2013-8 and \# 309079/2015-2. 


\section{Introduction}

Arboviruses are becoming increasingly widespread in a growing number of countries [Gubler, 2002, Liang et al., 2015]. While such a fast growth is likely to have many causes, the increasingly expansion of the associated vectors habitat's is certainly an important one-e.g. Lambrechts et al. [2010]. Indeed, two of the main and most effective vectors for such diseases, namely Aedes albopictus (the tiger mosquito) and Aedes aegypti (the yellow fever mosquito), are spreading well beyond tropical areas [Bhatt et al., 2013, Kraemer et al., 2015]. The consequences of this expansions is already evident: The south of Europe (Italy and southern France) has already documented cases of chikungunya [CDC, 2016], and in the US there is a growing number of dengue cases reported [Añez and Rios, 2013], and Zika is becoming a widespread concern[Samarasekera and Triunfol, 2016].

In large urban areas, the main vector for these arboviruses, Aedes aegypti, has a number of particular features: it is anthropophilic, lives only on urban or semi-urban areas, and it typically disperses no more than 500 meters from its birth place - cf. Honorio et al. [2003]. On the other hand, the existence of large urban transportation networks allow for long host movements. Thus, hosts can infect or become infected in regions that are geographically apart from their residence area, and circulation patters should play a major role in the disease dynamics. Such importance seems to be first pointed out by Adams and Kapan [2009], Cosner et al. [2009], Stoddard et al. [2009] in somewhat different contexts. Nevertheless, it seems apparent that this circulation effect will increase the population heterogeneity, and this typically favours epidemics [Dushoff and Levin, 1995, Hasibeder and Dye, 1988, Smith et al., 2004].

The study of multi-group models of epidemics dynamics can be traced back at least to the work of Lajmanovich and Yorke [1976], and subsequently in Nold [1980] and Hethcote and Yorke [1984]. Additional modelling work is subdivided populations can be found in Adler [1992], Andreasen and Christiansen [1989], Dushoff and Levin [1995], Hasibeder and Dye [1988] and also in the general analysis by Diekmann et al. [1990] formulated in terms of a continuous model. For meta-population models early analytical work can be traced back at least to the work of Arino and van den Driessche [2003].

The recognition of the importance of host movement and population heterogeneities lead to an increasing interest in the modelling literature for multi-group and meta-population models, and this has driven much recent work aiming to assess the impact of spatial heterogeneities and circulation patterns [Arino et al., 2012, Bichara et al., 2015, Bowong et al., 2013, Gatto et al., 2012, Padmanabha et al., 2012, Stoddard et al., 2013, Teurlai et al., 2012, Xiao and Zou, 2014]. Theoretical reviews on multi-group and meta-population models can de found in Thieme [2003] and in Arino [2009], respectively.

Most of earlier work seems to have focused on an specific framework, i.e. either on metapopulation or multi-group models. The latter might be thought as a fast sojourn limit of the former - e.g. Adams and Kapan [2009]. Nevertheless a systematic study of such asymptotic limit seems to be lacking. The aim of this work is three-fold: (i) to provide a systematic derivation of a multi-group model in the fast-sojourn limit such that the multi-group model parameters are obtained in terms of the parametrisation used in the meta-population model; (ii) understand how different degrees of heterogeneity impact on the disease dynamics; (iii) illustrate how such model can be parametrised with available data. 


\section{Methods}

The aim of this session is to present a consistent derivation of a multi-group model for urban environments. We begin by taking a detailed Lagrangian view for describing the disease dynamics. The urban environment will be denoted the City, and we assume that it is naturally divided into $n$ patches - which can be thought as districts and suburbs. The key assumptions in the model are as follows:

1. Each host individual is registered in some patch $i$, and we assume that individuals do not change their registered domiciles. Hence the total population that is registered in patch $i$, namely $N_{i}^{h}$, changes only as the result of birth and death. Further, there is no outward or inward flow-i.e. the outskirts are included in the city, and visitors are not accounted for.

2. The commuting time-scale within the city is fast when compared to either the demographic or epidemiological time-scales. The ratio of the latter time-scales to the former will denoted by the positive parameter $\epsilon \ll 1$. The commuting dynamics is also assumed to be time homogeneous.

3. Mosquitoes do not move.

4. In each patch, the disease dynamics is given by a Bailey-Dietz model, namely consists of two sub-models: SIR for hosts and SI for vectors [Bailey, 1975, Dietz, 1975].

Remark 2.1. We now comment on the assumptions above:

1. While this seems to be a simplifying assumption, we are not aware of any available data that could be used to model domicile registration dynamics. Moreover, disease notifications usually take domiciles for registration.

2. In large cities, a significant fraction of the population commutes daily-sometime through long distances. The typical average time for commuting ranges from 25 minutes to 50 minutes [Pereira and Schwanen, 2015], which is faster than the time-scales of both human, mosquito and disease dynamics. See Rocha et al. [2013], Souza [2014] for a discussion of the different time-scales and possible fast-slow scalings in arboviruses models. The assumption of time homogeneity is a simplification.

3. Most of urban mosquitoes - as is the case of Aedes aegypti are lazy flyers, and usually do not fly more than 500 meters away from the place they were born [Maciel-de Freitas and Lourenço-de Oliveira, 2009].

4. The Bailey-Dietz model has been extensively used to describe dengue dynamics [Esteva and Vargas, 1998, Nishiura, 2006], and it is the simplest model for such dynamics.

Let $N_{i j}^{h}(t)$ be the number of individuals registered in patch $i$ that are in patch $j$ at time $t$. These $N_{i j}$ are further subdivided in susceptible, infected and recovered, $S_{i j}^{h}, I_{i j}^{h}, R_{i j}^{h}$. Thus, we have the following aggregation relations:

$$
S_{i}^{h}=\sum_{j=1}^{n} S_{i j}^{h}, \quad I_{i}^{h}=\sum_{j=1}^{n} I_{i j}^{h}, \quad R_{i}^{h}=\sum_{j=1}^{n} R_{i j}^{h} \quad \text { and } \quad N_{i}^{h}=S_{i}^{h}+I_{i}^{h}+R_{i}^{h} .
$$


The total population is $N^{h}(t)=\sum_{i=1}^{n} N_{i}^{h}(t)$. Recalling that mosquitoes do not move from the residence patch, let us denote by $N^{v}$ the total mosquito population, which is also divided into patch populations $N_{i}^{v}, i=1, \ldots, n$. Analogously, this population of $N_{i}^{v}$ mosquitoes is further subdivided in $S_{i}^{v}$ and $I_{i}^{v}$. Under the assumptions discussed above, we are led to consider the following model with $3 n^{2}+2 n$ equations:

$$
\begin{aligned}
\dot{S}^{h}{ }_{i j} & =\mu_{i}^{h}\left(F_{i j}^{h}-S_{i j}^{h}\right)-\beta_{i j}^{h} \frac{S_{i j}^{h}}{\sum_{k=1}^{n} N_{k j}^{h}+A_{j}} I_{j}^{v}+\epsilon^{-1} \sum_{k=1}^{n} M_{j k}^{i} S_{i k}^{h} \\
\dot{I}^{h}{ }_{i j} & =\beta_{i j}^{h} \frac{S_{i j}^{h}}{\sum_{k=1}^{n} N_{k j}^{h}+A_{j}} I_{j}^{v}-\left(\mu_{i}^{h}+\gamma_{i}\right) I_{i j}^{h}+\epsilon^{-1} \sum_{k=1}^{n} M_{j k}^{i} I_{i k}^{h} \\
\dot{R}^{h}{ }_{i j} & =\gamma I_{i j}^{h}-\mu_{i}^{h} R_{i j}^{h}+\epsilon^{-1} \sum_{k=1}^{n} M_{j k}^{i} R_{i k}^{h} \\
\dot{S}^{v}{ }_{i} & =\mu_{i}^{v}\left(F_{i}^{v}-S_{i}^{v}\right)-S_{i}^{v} \sum_{k=1}^{n} \beta_{k i}^{v} \frac{I_{k i}^{h}}{\sum_{l=1}^{n} N_{l i}^{h}+A_{i}} \\
\dot{I}^{v}{ }_{i} & =S_{i}^{v} \sum_{k=1}^{n} \beta_{k i}^{v} \frac{I_{k i}^{h}}{\sum_{l=1}^{n} N_{l i}^{h}+A_{i}}-\mu_{i}^{v} I_{i}^{v} .
\end{aligned}
$$

System (2) is a Bailey-Dietz model for the population registered in $i$, which is visiting patch $j$ and with fast migration. The meaning of the remaining parameters is given in Table 1. Similar systems have been considered before for direct transmission diseases [Adams and Kapan, 2009, Arino and van den Driessche, 2003, Cosner et al., 2009].

\begin{tabular}{|c||l|}
\hline Parameters & Meaning \\
\hline \hline$\mu_{i}^{h}$ & Death rate for host in patch $i$ \\
\hline$\mu_{i}^{v}$ & Death rate for mosquitoes in patch $i$ \\
\hline$F_{i j}^{h}$ & $\begin{array}{l}\text { Carrying capacity associated to individuals } \\
\text { registered in patch } i \text { which frequently visit } \\
\text { patch } j\end{array}$ \\
\hline$F_{i}^{v}$ & Carrying capacity of mosquitoes in patch $i$ \\
\hline$\beta_{i j}^{h}$ & $\begin{array}{l}\text { Infectivity rates of host registered in } i \text { visit- } \\
\text { ing } j\end{array}$ \\
\hline$A_{i}$ & Alternative blood sources in patch $i$ \\
\hline$\gamma_{i}$ & Recovering rate of host in patch $i$ \\
\hline$\beta_{i j}^{v}$ & $\begin{array}{l}\text { Infectivity rates of vector in patch } j \text { interact- } \\
\text { ing with host from vector } i\end{array}$ \\
\hline$M_{j k}^{i}$ & $\begin{array}{l}\text { Migration rates matrix for host registered in } \\
\text { patch } i\end{array}$ \\
\hline
\end{tabular}

Table 1: Parameters in System (2) and their respective meaning. All rates are per-day. The remaining parameters are non-dimensional.

The carrying capacity for the hosts in patch $i$ is $F_{i}^{h}=\sum_{j=1}^{n} F_{i j}^{h}$.

Since the host population does not change their registered patch, the migration matrices $M^{i}$ 
must satisfy the following conditions:

$$
M_{j k}^{i} \geq 0, j \neq k, \quad M_{j j}^{i} \leq 0, \quad \text { and } \quad \mathbb{1}^{T} M^{i}=0,
$$

where $\mathbb{1}=(1, \cdots, 1)^{T}$, and the superscript $T$ denotes transpose.

Remark 2.2. Under the assumptions made, the equations for aggregated quantities $-N_{i}^{h}, S_{i}^{h}$, and $I_{i}^{h}$ - do not involve fast dynamic terms.

We now want to take advantage of the fast migration assumption to simplify System 2. In this vein, we will perform a multi-scaling reduction by assuming that the time scale $t$ of demographic and disease interactions are of order one or slower when compared to the fast time scale $t / \epsilon$ of human movement.

For the ensuing analysis it is convenient to rewrite System (2) using the variables $N_{i j}^{h}$ and $N_{i}^{v}$ as follows:

$$
\begin{aligned}
\dot{N}^{h}{ }_{i j} & =\mu_{i}^{h}\left(F_{i j}^{h}-N_{i j}^{h}\right)+\epsilon^{-1} \sum_{k=1}^{n} M_{j k}^{i} N_{i k}^{h} \\
\dot{N}^{v}{ }_{i} & =\mu_{i}^{v}\left(F_{i}^{v}-N_{i}^{v}\right) \\
\dot{S}^{h}{ }_{i j} & =\mu_{i}^{h}\left(F_{i j}^{h}-S_{i j}^{h}\right)-\beta_{i j}^{h} \frac{S_{i j}^{h}}{\sum_{k=1}^{n} N_{k j}^{h}+A_{j}} I_{j}^{v}+\epsilon^{-1} \sum_{k=1}^{n} M_{j k}^{i} S_{i k}^{h} \\
\dot{I}^{h}{ }_{i j} & =\beta_{i j} \frac{S_{i j}^{h}}{\sum_{k=1}^{n} N_{k j}^{h}+A_{j}} I_{j}^{v}-\left(\mu_{i}^{h}+\gamma_{i}\right) I_{i j}^{h}+\epsilon^{-1} \sum_{k=1}^{n} M_{j k}^{i} I_{i k}^{h} \\
\dot{I}^{v}{ }_{i} & =\left(N_{i}^{v}-I_{i}^{v}\right) \sum_{k=1}^{n} \beta_{k i}^{v} \frac{I_{k i}^{h}}{\sum_{l=1}^{n} N_{l i}^{h}+A_{i}}-\mu_{i}^{v} I_{i}^{v} .
\end{aligned}
$$

Recall that the null-space of $M^{i}$ has dimension at least one. It turns out that the simplicity of the reduced system will depend on the long-term behaviour of the fast migration dynamics. In order to obtain the simplest reduced system, we shall assume strong connectivity of the Lagrangian population dynamics in the urban environment. More precisely, we will add the following assumption:

Hypothesis 2.1. Let $\sigma\left(M^{i}\right)$ denote the spectrum of $M^{i}$. We will assume that

$$
\sigma\left(M^{i}\right) \subset \mathbb{C}^{-} \cup\{0\}, \quad i=1, \ldots, n,
$$

where $\mathbb{C}^{-}=\{z \in \mathbb{C} \mid \Re(z)<0\}$. We will also assume that zero is a simple eigenvalue for each $M^{i}$, i.e., that it has multiplicity one.

Remark 2.3. We observe that the strictly necessary assumption to derive a multi-group model using time-scale separation is

$$
\sigma\left(M^{i}\right) \subset \mathbb{C}^{-} \cup\{i \mathbb{R}\} \text { and } \sigma\left(M^{i}\right) \cap\{0\} \neq \emptyset, \quad i=1, \ldots, n,
$$

with zero not being a defective eigenvalue.

Regarding the assumptions in Hypothesis 2.1, we observe that

1. The absence of pure imaginary eigenvalues eliminates the need of homogenising the urban dynamics. This is a simplifying assumption. 
2. The simplicity of the zero eigenvalue of $M^{i}$ is equivalent to strong connectivity of the Lagrangian dynamics of the population registered at patch $i$. For daily commuting networks this is a reasonable assumption, and it is satisfied if all commuters registered in patch $i$ depart from and return to patch $i$.

In order to obtain a multi-scaling reduction, we introduce the fast time variable $\epsilon \tau=t$, and perform an asymptotic expansion using the method of multiple scales. For the dynamics of hosts we write the following composite asymptotic expansion:

$$
\begin{aligned}
N_{i j}^{h}(t) & =\tilde{N}^{h}{ }_{i j}(\tau)+\hat{N}^{h}{ }_{i j}(t)+\mathcal{O}(\epsilon) \\
S_{i j}^{h}(t) & =\tilde{S}^{h}{ }_{i j}(\tau)+\hat{S}^{h}{ }_{i j}(t)+\mathcal{O}(\epsilon) \\
I^{h}(t)_{i j} & =\tilde{I}^{h}{ }_{i j}(\tau)+\hat{I}^{h}{ }_{i j}(t)+\mathcal{O}(\epsilon) .
\end{aligned}
$$

Let $V_{i}$ denote the null-space of $M^{i}$. Given an initial condition $S_{i j}^{h}(0)$ and $I_{i j}^{h}(0)$ we write

$$
S_{i j}^{h}(0)=\tilde{S}_{i j}(0)+\hat{S}^{h}{ }_{i j}(0), \quad I^{h}(0)=\tilde{I}^{h}{ }_{i j}(0)+\hat{I}^{h}{ }_{i j}(0)
$$

where $\hat{S}^{h}{ }_{i} \in V_{i}$, and $\tilde{S}^{h}{ }_{i} \in V_{i}^{\perp}$, and likewise for $I_{i}^{h}(0)$-and hence of $N_{i}^{h}(0)$. The tilde variables obey the following equations in the fast time variable $\tau$ :

$$
\begin{aligned}
\frac{d}{d \tau} \tilde{N}^{h}{ }_{i j} & =\sum_{k=1}^{n} M_{j k}^{i} \tilde{N}^{h}{ }_{i k} \\
\frac{d}{d \tau} \tilde{S}^{h}{ }_{i j} & =\sum_{k=1}^{n} M_{j k}^{i} \tilde{S}^{h}{ }_{i k} \\
\frac{d}{d \tau} \tilde{I}^{h}{ }_{i j} & =\sum_{k=1}^{n} M_{j k}^{i} \tilde{I}^{h}{ }_{i k}
\end{aligned}
$$

Remark 2.4. All systems above are instances of the following system:

$$
\frac{d}{d \tau} X^{(i)}(\tau)=M^{i} X^{(i)}
$$

with solution given by

$$
X^{(i)}(\tau)=\exp \left(\tau M^{i}\right) X^{(i)}(0) .
$$

In addition, the conditions in (3) ensure that $M^{i}$ is a Metzler matrix, and hence $\exp \left(\tau M^{i}\right)$ is a non-negative matrix [Berman and Plemmons, 1979]. Also, since $\mathbb{1}^{T} M^{i}=0^{T}$, we have that $\mathbb{1}^{T} \exp \left(\tau M^{i}\right)=\mathbb{1}^{T}$, and therefore it is a stochastic matrix-cf. Norris [1997].

In view of Hypothesis 2.1, we have that there exists a matrix $C^{i}$ such that $\lim _{\tau \rightarrow \infty} \exp \left(\tau M^{i}\right)=$ $C^{i}$ [Teschl, 2012], which is then stochastic. Moreover, $\sigma\left(C^{i}\right)=\{0,1\}$, with multiplicities $n-1$ and 1, respectively. Therefore $C^{i}$ is a rank one matrix, i.e, we have-cf. Horn and Johnson [1990]-that

$$
C^{i}=c_{i} \mathbb{1}^{T}, \text { with } c_{i}=\left(c_{i 1}, \ldots, c_{i n}\right)^{T}, \quad c_{i j} \geq 0, \quad \sum_{j=1}^{n} c_{i j}=1 .
$$

Thus, from the matrices $C^{i}$ we obtain the circulation matrix $c$, that is a stochastic matrix. 
In view of the preceding, we obtain

$$
\lim _{\tau \rightarrow \infty}\left(\tilde{N}^{h}(\tau), \tilde{S}^{h}(\tau), \tilde{I}^{h}(\tau)\right)=0
$$

and that

$$
\begin{aligned}
\hat{N}^{h}{ }_{i j} & =\sum_{k=1}^{n} C_{j k}^{i} \tilde{N}^{h}{ }_{i k} \\
\hat{S}^{h} & =\sum_{k=1}^{n} C_{j k}^{i} \tilde{S}^{h}{ }_{i k} \\
\hat{I}^{h} & =\sum_{k=1}^{n} C_{j k}^{i} \tilde{I}^{h}{ }_{i k},
\end{aligned}
$$

since $\hat{\star}_{i}^{h} \in V^{i}, \star=N, S, I$.

We now abuse language and drop the hat symbols, and consider the aggregated variables in order to obtain the following system in slow time variable $t$ :

$$
\begin{aligned}
\dot{N}^{h}{ }_{i} & =\mu_{i}^{h}\left(F_{i}^{h}-N_{i}^{h}\right) \\
\dot{N}^{v}{ }_{i} & =\mu_{i}^{v}\left(F_{i}^{v}-N_{i}^{v}\right) \\
\dot{S}^{h}{ }_{i} & =\mu_{i}^{h}\left(F_{i}^{h}-S_{i}^{h}\right)-\sum_{j=1}^{n} \beta_{i j}^{h} \frac{S_{i j}^{h}}{\sum_{k=1}^{n} N_{k j}^{h}+A_{j}} I_{j}^{v} \\
\dot{I}_{i} & =\sum_{j=1}^{n} \beta_{i j}^{h} \frac{S_{i j}^{h}}{\sum_{k=1}^{n} N_{k j}^{h}+A_{j}} I_{j}^{v}-\left(\mu_{i}^{h}+\gamma_{i}\right) I_{i j}^{h} \\
\dot{I}^{v}{ }_{i} & =\sum_{j=1}^{n} \beta_{j i}^{v} \frac{I_{j i}^{h}}{\sum_{k=1}^{n} N_{k i}^{h}+A_{i}}\left(N_{i}^{v}-I_{i}^{v}\right)-\mu_{i}^{v} I_{i}^{v}
\end{aligned}
$$

supplemented by algebraic conditions given by (7) (with hats dropped). Notice that System (8) is not entirely written in terms of the aggregate variables for each registration patch $i$. In order to achieve this goal, we use the algebraic conditions above, and the circulation matrix $c$ described in Remark 2.4 to obtain that:

$$
S_{i j}^{h}=\sum_{k=1}^{n} C_{j k}^{i} S_{i k}^{h}=\sum_{k=1}^{n} c_{i j} S_{i k}^{h}=c_{i j} \sum_{k=1}^{n} S_{i k}^{h}=c_{i j} S_{i}^{h} .
$$

Analogously

$$
I_{i j}^{h}=c_{i j} I_{i}^{h} \quad \text { and } \quad N_{i j}^{h}=c_{i j} N_{i}^{h} .
$$

Notice that the last constraint yields also a compatibility condition for the host carrying capacities, namely, they must satisfy:

$$
F_{i j}^{h}=c_{i j} F_{i}^{h} .
$$

Remark 2.5. In practical applications, the information that is typically available are the carrying capacities of the patches, namely $F_{i}$. The parameters $F_{i j}$ are then given as a fraction of $F_{i}$, and a very natural choice for such a fraction is the average proportion of individuals registered in patch $i$ that usually visit patch $j$. This leads to Equation (9). 
We now combine all these results in order to arrive at the following fully aggregated system:

$$
\begin{aligned}
\dot{N}^{h}{ }_{i} & =\mu_{i}^{h}\left(F_{i}^{h}-N_{i}^{h}\right) \\
\dot{N}^{v}{ }_{i} & =\mu_{i}^{v}\left(F_{i}^{v}-N_{i}^{v}\right) \\
\dot{S}^{h}{ }_{i} & =\mu_{i}^{h}\left(F_{i}^{h}-S_{i}^{h}\right)-S_{i}^{h} \sum_{j=1}^{n} \beta_{i j}^{h} c_{i j} \frac{I_{j}^{v}}{\sum_{k=1}^{n} c_{k j} N_{k}^{h}+A_{j}} \\
\dot{I}^{h}{ }_{i} & =S_{i}^{h} \sum_{j=1}^{n} \beta_{i j}^{h} c_{i j} \frac{I_{j}^{v}}{\sum_{k=1}^{n} c_{k j} N_{k}^{h}+A_{j}}-\left(\mu_{i}^{h}+\gamma_{i}\right) I_{i}^{h} \\
\dot{I}^{v}{ }_{i} & =\sum_{k=1}^{n} \beta_{k i}^{v} c_{k i} \frac{I_{k}^{h}}{\sum_{l=1}^{n} c_{l i} N_{l}^{h}+A_{i}}\left(N_{i}^{v}-I_{i}^{v}\right)-\mu_{i}^{v} I_{i}^{v}
\end{aligned}
$$

We now want to write System (10) in matrix form, and will need to introduce some notation: given a vector $u \in \mathbb{R}^{n}$, we will write $\operatorname{diag}(u)$ for the diagonal $n \times n$ matrix, whose main diagonal

is $u$; given two matrices with the same dimensions, $M_{1}$ and $M_{2}$, we will write $M_{1} \circ M_{2}$ for the Hadamard product of $M_{1}$ and $M_{2}$ - the entrywise product.

Also, we let

$$
C=\left(c_{i j}\right), \quad B^{h}=\left(\beta_{i j}^{h}\right), \quad B^{v}=\left(\beta_{i j}^{v}\right) .
$$

Then we may rewrite System (10) as

$$
\begin{aligned}
\dot{N}^{h} & =\operatorname{diag}\left(\mu^{h}\right)\left(F^{h}-N^{h}\right) \\
\dot{N}^{v} & =\operatorname{diag}\left(\mu^{v}\right)\left(F^{v}-N^{v}\right) \\
\dot{S^{h}} & =\operatorname{diag}\left(\mu^{h}\right)\left(F^{h}-S^{h}\right)-\operatorname{diag}\left(S^{h}\right)\left(B^{h} \circ C\right) \operatorname{diag}^{-1}\left(C^{T} N^{h}+A\right) I^{v} \\
\dot{I^{h}} & =\operatorname{diag}\left(S^{h}\right)\left(B^{h} \circ C\right) \operatorname{diag}^{-1}\left(C^{T} N^{h}+A\right) I^{v}-\operatorname{diag}\left(\mu^{h}+\gamma\right) I^{h} \\
\dot{I^{v}} & =\operatorname{diag}\left(N^{v}-I^{v}\right) \operatorname{diag}^{-1}\left(C^{T} N^{h}+A\right)\left(B^{v} \circ C\right)^{T} I^{h}-\operatorname{diag}\left(\mu^{v}\right) I^{v}
\end{aligned}
$$

Several variants of System 10 have been considered before - e.g. Adams and Kapan [2009], Alvim et al. [2013].

\section{Theory and Computations}

\subsection{Preliminaries}

System (11) is a special case of a class of models studied in Iggidr et al. [2016], provided we make some assumptions: $B^{h}, B^{v}$ are positive matrices; $C^{T} u+A$ is a positive vector, whenever $u$ is a positive vector. The latter is a non-zero mosquito blood source (either human or alternative) assumption, and it is satisfied, for instance, if $C$ is irreducible or has positive diagonal-however, these are not necessary conditions.

Following Iggidr et al. [2016], we denote the graph associated to a matrix $M$ by $\Gamma(M)$, and we introduce the host-vector contact network as $\Gamma(\mathcal{M})$, where $\mathcal{M}$ is given by

$$
\mathcal{M}=\left(\begin{array}{cc}
0 & B^{h} \circ C \\
\left(B^{v} \circ C\right)^{T} & 0
\end{array}\right) .
$$


Notice that, since $B^{h}$ an $B^{v}$ are positive, we have that $\Gamma(\mathcal{M})$ is the same as $\Gamma\left(\mathcal{M}_{0}\right)$, with

$$
\mathcal{M}_{0}=\left(\begin{array}{cc}
0 & C \\
C^{T} & 0
\end{array}\right) .
$$

If $\mathcal{M}$ is irreducible then a whole class of multi-group SIR-SI models, which includes System 10, satisfies what is known as the sharp threshold property - as defined in Shuai and van den Driessche [2013]. Namely, we have that

1. For all parameters there is always a feasible equilibrium with null infected hosts and vectorsthe so called disease free equilibrium (DFE).

2. If $R_{0} \leq 1$ then the only feasible equilibrium is the DFE, and it is globally asymptotically stable.

3. If $R_{0}>1$ then there is also an unique endemic equilibrium (EE), and it is globally asymptotically stable for initial conditions with non-vanishing infected hosts or vectors.

For a proof of these results see Iggidr et al. [2016].

Remark 3.1. As observed in Iggidr et al. [2016, Remark 2.3], the irreducibility of $C$ is neither necessary nor sufficient for the irreducibility of $\mathcal{M}$ (or equivalently of $\mathcal{M}_{0}$ ). Indeed, $\mathcal{M}_{0}$ is irreducible if, and only if, both $C C^{T}$ and $C^{T} C$ are irreducible and if the interior of the non-negative orthant in $\mathbb{R}^{n}$ is invariant through $C$-cf. Iggidr et al. [2016, Proposition 2.1].

\subsection{Basic reproduction ratio $-R_{0}$}

Following Diekmann et al. [1990], van den Driessche and Watmough [2002], we observe that the next-generation matrix for System (10) is

$$
\mathcal{N}=\left(\begin{array}{cc}
0 & \mathcal{N}_{12} \\
\mathcal{N}_{21} & 0
\end{array}\right)
$$

with

$$
\mathcal{N}_{12}=\operatorname{diag}\left(\mu^{h}+\gamma\right)^{-1} \operatorname{diag}\left(F^{h}\right)\left(B^{h} \circ C\right) \operatorname{diag}\left(C^{T} F^{h}+A\right)^{-1},
$$

and

$$
\mathcal{N}_{21}=\operatorname{diag}\left(\mu^{v}\right)^{-1} \operatorname{diag}\left(F^{v}\right) \operatorname{diag}\left(C^{T} F^{h}+A\right)^{-1}\left(B^{v} \circ C\right)^{T} .
$$

In this framework, the spectral radius of $\mathcal{N}$ yields the basic reproduction rate, namely:

$$
R_{0}=\rho(\mathcal{N})
$$

In what follows, we are interested in study the behaviour of $R_{0}$ with respect to the different heterogeneities present in this model. In this vein, we introduce

$$
\bar{N}=\mathbb{1}^{T} C^{T} F^{h}=\mathbb{1}^{T} F^{h}, \quad \bar{V}=\mathbb{1}^{T} F^{v} \quad \text { and } \quad \bar{a}=\mathbb{1}^{T} A
$$

for the total host, vector populations and the total alternative blood sources, respectively. We also introduce the average values of the different parameters

$$
\mu_{h}=\frac{1}{n} \mathbb{1}^{T} \mu^{h}, \quad \mu_{v}=\frac{1}{n} \mathbb{1}^{T} \mu^{v}, \quad \text { and } \quad \gamma_{h}=\frac{1}{n} \mathbb{1}^{T} \gamma
$$


With this notation, we introduce the heterogeneity parameter vectors:

$$
\operatorname{diag}\left(\mu^{h}+\gamma\right)=\left(\mu_{h}+\gamma_{h}\right) \operatorname{diag}(\hat{\omega}), \quad \operatorname{diag}\left(\mu^{v}\right)=\mu_{v} \operatorname{diag}\left(\hat{\mu}^{v}\right), \quad \operatorname{diag}\left(C^{T} F^{h}+A\right)=\frac{\bar{N}+\bar{a}}{n} \operatorname{diag}(\hat{\zeta}),
$$

the host and vector densities

$$
\operatorname{diag}\left(F^{h}\right)=\frac{\bar{N}}{n} \operatorname{diag}\left(\hat{F}^{h}\right), \quad \operatorname{diag}\left(F^{v}\right)=\frac{\bar{V}}{n} \operatorname{diag}\left(\hat{F}^{v}\right),
$$

and the infectivities heterogeneity matrices

$$
B^{h}=b \beta_{h} \hat{B}, \quad B^{v}=b \beta_{v} \hat{B}
$$

where $b$ is the average number of bites per mosquito, per day, and $\beta^{h}$ and $\beta^{v}$ are representative values of the host and vector infectivities. Notice that in Equation 12 we are assuming that infectivities can be different for host and vector, but that inhomogeneities are the same. This seems a reasonable assumption, if one takes the view that $\hat{B}$ is, as a matter of fact, modulating the biting behaviour of mosquitoes in different host-vector encounters.

We now give a convenient alternative expression for $R_{0}$ :

Theorem 3.1. Let

$$
\widetilde{R_{0}}=\left[\frac{b^{2} \beta_{h} \beta_{v}}{\left(\mu_{h}+\gamma_{h}\right) \mu_{v}} \frac{\bar{N}}{\bar{N}+\bar{a}} \frac{\bar{V}}{\bar{N}+\bar{a}}\right]^{1 / 2}
$$

be the basic reproduction ration for the single-patch model associated to System 10, and let

$$
L=\operatorname{diag}(\hat{\omega})^{-1 / 2} \operatorname{diag}\left(\hat{F}^{h}\right)^{1 / 2}(\hat{B} \circ C) \operatorname{diag}\left(\hat{\mu}^{v}\right)^{-1 / 2} \operatorname{diag}\left(\hat{F}^{v}\right)^{1 / 2} \operatorname{diag}(\hat{\zeta})^{-1},
$$

Then

$$
R_{0}=\widetilde{R_{0}} \sigma_{1}(L)
$$

where $\sigma_{1}(L)$ denotes the largest singular value of $L$.

Proof. Let

$$
\begin{aligned}
& P=\left(\begin{array}{cc}
P_{1} & 0 \\
0 & P_{2}
\end{array}\right) \\
& P_{1}=\left(b \beta_{h}\right)^{-1 / 2} \operatorname{diag}\left(\mu^{h}+\gamma\right)^{1 / 2} \operatorname{diag}\left(F^{h}\right)^{-1 / 2} \\
& P_{2}=\left(b \beta_{v}\right)^{-1 / 2} \operatorname{diag}\left(\mu^{v}\right)^{1 / 2} \operatorname{diag}\left(F^{v}\right)^{-1 / 2}
\end{aligned}
$$

Then $P$ is non-singular and a direct calculation shows that

$$
P \mathcal{N} P^{-1}=\widetilde{R_{0}}\left(\begin{array}{cc}
0 & L \\
L^{T} & 0
\end{array}\right)
$$

from which the result follows.

Remark 3.2. Notice that Equation (15) expresses $R_{0}$ as product of the single-patch $\widetilde{R_{0}}$ - which can be seen as a uniform $R_{0}$, i.e., the $R_{0}$ that would be obtained if we disregard all heterogeneitiesand a heterogeneous correction factor giving by the largest singular value of $L$ given. The matrix $L$ encodes, as far as the basic reproduction ratio $R_{0}$ is concerned, all the heterogeneities of the model: 
$\hat{\omega}$ non-uniformity in human biology concerning both longevity and recovering capabilities;

$\hat{\mu^{v}}$ non-uniformity in vector biology concerning longevity;

$\hat{B}$ interaction of origin-destiny patches in the local infectivity;

$\hat{F}^{h}$ non-uniformity of human occupation of the City;

$\hat{F}^{v}$ non-uniformity of vector occupation of the region;

$\hat{\zeta}$ non-uniformity in the distribution of mean effective sources of blood meals;

$C$ circulation matrix.

Remark 3.3. We point out that, if a particular feature is homogeneous, the corresponding hatted vector will be $\mathbb{1}$, and an uniform $\hat{B}$ will be given by $\mathbb{1} \otimes \mathbb{1}$. In this framework, different levels of modelling detail can be contemplated:

Fully Detailed This requires full description of human and mosquitoes biological parameters (including the recovering rate) in all patches, epidemiological infectivity parameters, host and vector density occupation, mean blood source distribution, and circulation pattern.

Biologically Homogeneous (BH) In the absence of further information, one might use literature values for the host and vector biological parameters. In this case, we have $\hat{\omega}=\hat{\mu}^{v}=\mathbb{1}$.

Epidemiologically and Biologically Homogeneous (EBH) In this case, we take $\hat{B}=\mathbb{1} \otimes \mathbb{1}$, and the heterogeneity of the model comes only from the density distribution of host and vectors, and circulation patterns.

Circulation Only (CO) In this coarser parametrisation scope, one assume also that $\hat{F}^{h}=\hat{F}^{v}=$ $\mathbb{1}$, and hence the only heterogeneity is due to circulation.

The choice of the modelling scope will depend on the data available to parametrise the models. An approximation of the circulation matrix $C$ can be usually obtained using data available from transport authorities. The values of $\hat{F}^{h}$ are usually available from census information of the city, $\hat{F}^{v}$ from mosquito infestation surveys that are usually periodically performed in cities where such diseases are of concern - and this also the case for $\hat{\zeta}$. On the other hand, $\hat{\omega}$ will be only available if sufficient medical data is available, and analogously $\hat{\mu}^{v}$ will be only available if there is sufficient entomological data in the region. Finally, the parametrisation of the infectivity matrix is always a challenge in epidemiological modelling [Eubank et al., 2004, Lawson and Leimich, 2000, Martens et al., 1995].

In this work, we will focus on the EBH level of detail, although we also include some examples in the BH scope. In the latter case, we offer a rationale to construct $\hat{B}$ from a reduced set of parameters. Namely, we consider two vectors $\eta$ and $\xi$, and define

$$
\hat{B}=\eta \otimes \xi
$$

In this framework, $\eta$ can be thought as a scaling vector taking into account the socio-economic behaviour of the host (for instance) based on its patch of registration. This might include the fact that hosts from well-informed patches will likely use repellent, appropriate clothes and avoid risky behaviour. On the other hand, $\xi$ is a scaling vector taking into account either the environment at 
the visited location or the local biting behaviour of mosquitoes. Finally, we point out that a similar result for a system with a different infection force, and under additional hypothesis, can be found in Alvim et al. [2013].

We want to finish this section showing that, if we are in the BH modelling scope, and if there are no alternative blood sources, then one always have $\widetilde{R_{0}} \leq R_{0}$. Moreover, under the CO modelling scope, equality holds if $C$ is bi-stochastic.

Theorem 3.2. Assume that $A=0, \hat{\omega}=\hat{\mu}^{v}=\mathbb{1}$. Then we always have $R_{0} \geq \widetilde{R_{0}}$. Moreover, if $\hat{F}^{h}=\hat{F}^{v}=\mathbb{1}$, and $\hat{B}=\mathbb{1} \otimes \mathbb{1}$ then equality holds if $C$ is bi-stochastic.

Proof. For a non-negative vector $v$, let us write $v^{1 / 2}$ for the entry-wise square-root of $v$, and $v^{T / 2}$ for its transpose. Then a direct calculation shows that

$$
\hat{F}^{T / 2} L=\hat{F}^{T / 2} \text {. }
$$

Taking inner products we arrive at

$$
\begin{aligned}
\left\|\hat{F}^{v^{1 / 2}}\right\|_{2}^{2} & =\left\|\hat{F}^{v^{1 / 2}}\right\|_{2}\left\|L^{T} \hat{F}^{h^{1 / 2}}\right\|_{2} \\
& \leq\left\|\hat{F}^{v^{1 / 2}}\right\|_{2}\left\|\hat{F}^{h^{1 / 2}}\right\|_{2}\left\|L^{T}\right\|_{2} \\
& =\leq\left\|\hat{F}^{v^{1 / 2}}\right\|_{2}\left\|\hat{F}^{1 / 2}\right\|_{2} \sigma_{1}(L)
\end{aligned}
$$

Now, we observe that

$$
\left\|\hat{F}^{h^{1 / 2}}\right\|_{2}=\left\|\hat{F}^{h}\right\|_{1}=n \quad \text { and } \quad\left\|\hat{F}^{v^{1 / 2}}\right\|_{2}=\left\|\hat{F}^{v}\right\|_{1}=n
$$

Hence, we conclude that

$$
\sigma_{1}(L) \geq 1
$$

and we obtain that $R_{0} \geq \widetilde{R_{0}}$ as claimed.

If $\hat{F}^{h}=\hat{F}^{v}=\mathbb{1}$, and if $C$ is bi-stochastic, then $L=C$ and, since $\sigma_{1}(C)=1$, the result follows.

\subsection{Attacking ratio}

In traditional empirical epidemiology, one of the ways to estimate $R_{0}$ is to measure the so-called attack ratio at the beginning of the epidemic, and then determine the exponential growth rate. If one has a simple model such that an expression linking $R_{0}$ to the most unstable eigenvalue is available, then then one can translate a estimative of the disease growth rate into an estimative of $R_{0}$. It turns out that the model presented here allows for such an expression. We present a proof within a very simplified setting, but we point out that it can be easily extended to the $\mathrm{BH}$ modelling scope.

Proposition 3.1. Let us assume that we are in the EBH modelling scope-i.e. $\hat{\omega}=\hat{\mu}^{v}=\mathbb{1}$ and $\hat{B}=\mathbb{1} \otimes \mathbb{1}$-and assume further that $\hat{\zeta}=\mathbb{1}$. Let

$$
c=\frac{\mu_{v}+\mu_{h}+\gamma_{h}}{2}, d=\mu_{v}\left(\mu_{h}+\gamma_{h}\right)
$$

Then the largest eigenvalue of the infected subsystem at the DFE is given by

$$
-c+\sqrt{c^{2}+d\left(R_{0}^{2}-1\right)}
$$

The proof is presented in AppendixA. 


\subsection{Local $R_{0}$ and curfew}

If the patches were isolated, then we would have $C=I$, and in this case System (10) becomes $n$ disconnected Bailey-Ditz models. In this case, each patch would have its own local $R_{0}$, and each dynamics would evolve separately. In this situation, the natural question as to what happens when circulation is "weak", i.e., if the matrix $C$ is sufficiently close to the identity. Within this view, an appropriate measure of circulation strength might me given by $\|C-I\|$, where $\|\cdot\|$ is some given matrix norm. We will not digress on the possible choices of norm, but will instead introduce the curfew path. Namely, if $C$ is a circulation matrix, we define

$$
\mathcal{C}_{s}=(1-s) C+s I, \quad s \in[0,1]
$$

and we will say that $s$ is the curfew strength. We have the following result:

Proposition 3.2. Assume that we are in the BH modelling scope. Then following holds:

1. If $C=I$, then each patch evolves independently with local basic reproductive number given by

$$
R_{0}^{\mathrm{loc}, i}=\widetilde{R_{0}} \sqrt{\frac{\bar{N} F_{i}^{v}}{\bar{V} F_{i}^{h}}} \hat{B}_{i i}
$$

2. If $C=I$, then we have

$$
R_{0}=\max _{i=1, \ldots, n} R_{0}^{\text {loc }, i}
$$

with $R_{0}^{\text {loc, } i}$ given above.

3. Consider the curfew path given by Equation (18), and let $R_{0}(s)$ be the basic reproduction ratio, when the circulation matrix is given by $\mathcal{C}_{s}$. Then

$$
\lim _{s \rightarrow 1} R_{0}(s)=\max _{i=1, \ldots, n} R_{0}^{\text {loc }, i}
$$

Proof. 1 follows from the fact that System (10) is diagonal when $C=I$, and the expression follows from a straight direct computation. 2 follows immediately from the fact that when $C=I$, then $L$ is also diagonal, with entries given $\sqrt{\frac{\bar{N} F_{i}^{v}}{\bar{V} F_{i}^{h}}}$. Finally 3 is a consequence of the continuity of singular values with respect to matrix entries.

Remark 3.4. Notice that any patch that connects the matrix $C$ to the identity in the set of row stochastic matrices can be used, provided it contains only irreducible matrices. The path given by Equation (18) has the advantage of being both simple and to preserve the network topology for $0 \leq s<1$. Notice also that Proposition 3.2 shows that maximum of $R_{0}^{\text {loc }, i}$ dominates the behaviour of $R_{0}$ if the circulation is weak. In particular, if this maximum is greater than one, then reducing the circulation will eventually lead to a system with $R_{0}>1$-example of this behaviour have been found recently though simulations. We also point out that local $R_{0}$ haven been already considered in Alvim et al. [2013], Arino and van den Driessche [2003]. 


\subsection{Control}

Assume that we are able to slay a fraction $0 \leq \alpha<1$ of mosquitoes. The next result gives a crude lower bound on the reproduction numbers that can be achieved in such way.

Proposition 3.3. Let

$$
0 \leq \alpha \leq \min _{i=1, \ldots, n} F_{i}^{v}
$$

and let $\bar{R}_{0}(\alpha)$ the reproductive number obtained after slaying this fraction in some region $i_{0}$. Then $\bar{R}_{0}(\alpha)$ is a non-increasing function and we have

$$
\left(1-\frac{\alpha}{\hat{F}_{i_{0}}^{v}}\right)^{1 / 2} R_{0} \leq \bar{R}_{0}(\alpha) .
$$

Proof. A direct calculation shows that

$$
\bar{R}_{0}(\alpha)=(1-\alpha)^{1 / 2} \widetilde{R_{0}} \sigma_{1}(\bar{L}), \quad \bar{L}=L \operatorname{diag}\left(\bar{F}^{v}\right)^{1 / 2},
$$

where

$$
\bar{F}^{v}=\frac{1}{1-\alpha}\left(1,1, \ldots, 1-\frac{\alpha}{\hat{F}_{i_{0}}^{v}}, \ldots, 1\right) .
$$

Using well-know bounds for the singular values of the product of two matrices [Horn and Johnson, 1991], namely

$$
\sigma_{1}(A) \sigma_{n}(B) \leq \sigma_{1}(A B) \leq \sigma_{1}(A) \sigma_{1}(B)
$$

we obtain that

$$
\left(1-\frac{\alpha}{F_{i_{0}}^{v}}\right)^{1 / 2} R_{0} \leq \bar{R}_{0}(\alpha) \leq R_{0} .
$$

This already yields the claimed lower bound. On the other hand, applying the upper bound to $\bar{R}_{0}(\alpha)$ instead of $R_{0}$ shows that $\bar{R}_{0}(\alpha)$ is non-increasing.

\section{Results and discussion}

\subsection{A hypothetical City}

The purpose of these examples is to illustrate how the epidemiological dynamics in a urban environment are sensitive to human circulation. Consider a hypothetical city divided into three regions:

The city centre (Region 0) is where a large a number of inhabitants holds their jobs. It receives a large flow from both Region 1 and 2; the outflow of the city centre is very small. Region 1 has a significant outflow to the city centre, with a significant fraction of the inhabitants staying within the region. Also, there is some small outflow to Region 2. Finally, Region 2 has a large outflow to the city centre, and some outflow to Region 1. For the fraction of the population staying in Region 3, we take two different scenarios: one where half of the population stays, and another where about one third stays.

We consider two circulations matrices that fit the rationale above.

We also take $A=0$, which is the correct modelling assumption for Aedes aegypti for instance. 


$$
C_{1}=\left(\begin{array}{ccc}
0.9 & 0.05 & 0.05 \\
0.55 & 0.44 & 0.01 \\
0.49 & 0.01 & 0.5
\end{array}\right) \| C_{2}=\left(\begin{array}{ccc}
0.9 & 0.05 & 0.05 \\
0.5 & 0.45 & 0.05 \\
0.55 & 0.1 & 0.35
\end{array}\right)
$$

Table 2: Two examples of circulation matrices which are consistent with the rationale of the hypothetical city described in the text.

For the biological and epidemiological parameters, we follow Nishiura [2006] and take:

$$
\beta_{v}=0.4, \quad \beta_{h}=0.4, \mu_{h}=0.0000457, \mu_{v}=0.25, \quad \gamma_{h}=0.167 \text {. }
$$

We then take the vector to host-ratio - the ratio $\frac{\bar{V}}{\bar{N}}$-such that $\widetilde{R_{0}}=1$. Therefore, if we use a single patch model so as to disregard both circulation patterns and population heterogeneities, we would conclude that we should be in a non-epidemic situation - although at a critical condition. In the following we investigate the influence of non-uniformity and circulation within the $\mathrm{BH}$ and EBH modelling scopes.

\subsubsection{Non-uniformities and $R_{0}$}

We begin by considering the effects of host distribution heterogeneities, for a uniform distribution of vectors. More specifically, we allow both host occupancy varies from 0.05 to 0.9 in the City Centre, and Region 1 -i.e. $1 / 3 \hat{F_{h, i}}$ and $1 / 3 \hat{F_{h, 2}}$. Hence the corresponding occupancy in Region 2 is $1 / 3 \hat{F}_{h, 3}=1-1 / 3 \hat{F}_{h, 1}-1 / 3 \hat{F}_{h, 2}$. These results are given in Figure 1 . Notice also that $A=0$, and that we are either in the $\mathrm{BH}$ or $\mathrm{EBH}$ modelling scope. Hence Theorem 3.2 applies and we always have $R_{0} \geq 1$.

The dual problem of considering the effects of the vector distribution given a uniform distribution of hosts was also investigated and the results are displayed in Figure 2.

\subsubsection{Anomalous $R_{0}$ configurations and curfew}

Definition 4.1. We will say that a configuration is anomalous if we have either

1. $R_{0}<\min _{i} R_{0}^{\mathrm{loc}, i}$;

2. $\max _{i} R_{0}^{\mathrm{loc}, i}<R_{0}$

Remark 4.1. Notice that if we have $F^{h}=F^{v}$, then all local $R_{0}$ are equal. Otherwise, we necessarily have that

$$
\min _{i} R_{0}^{\mathrm{loc}, i}<\widetilde{R_{0}}<\max _{i} R_{0}^{\mathrm{loc}, i}
$$

Thus, when $A=0$ and we are in the BH modelling scope, Theorem 3.2 applies, and situation (1) cannot happen.

In the computations presented in Figures 1 and 2 the fraction of anomalous configuration varied between 0 and 0.08 . The existence of such anomalous configuration leads to the situation described in the next result: 


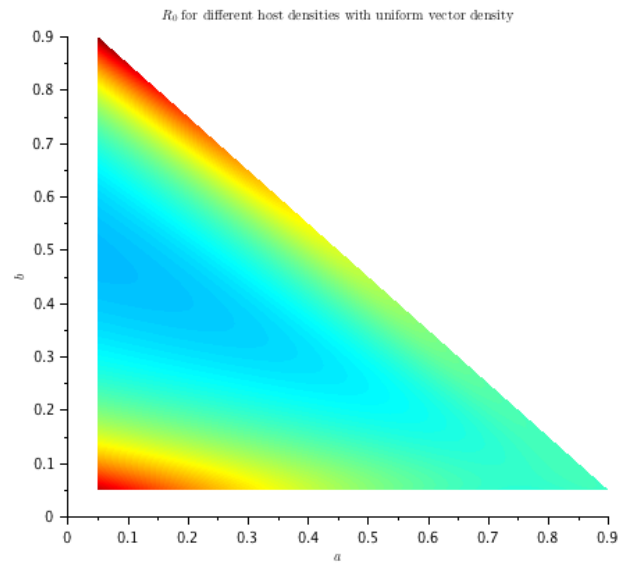

(a) $C_{1}$

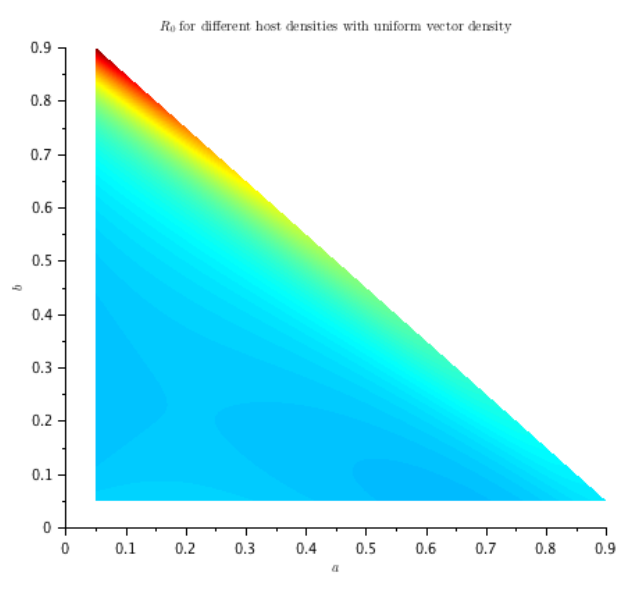

(c) $C_{1}$
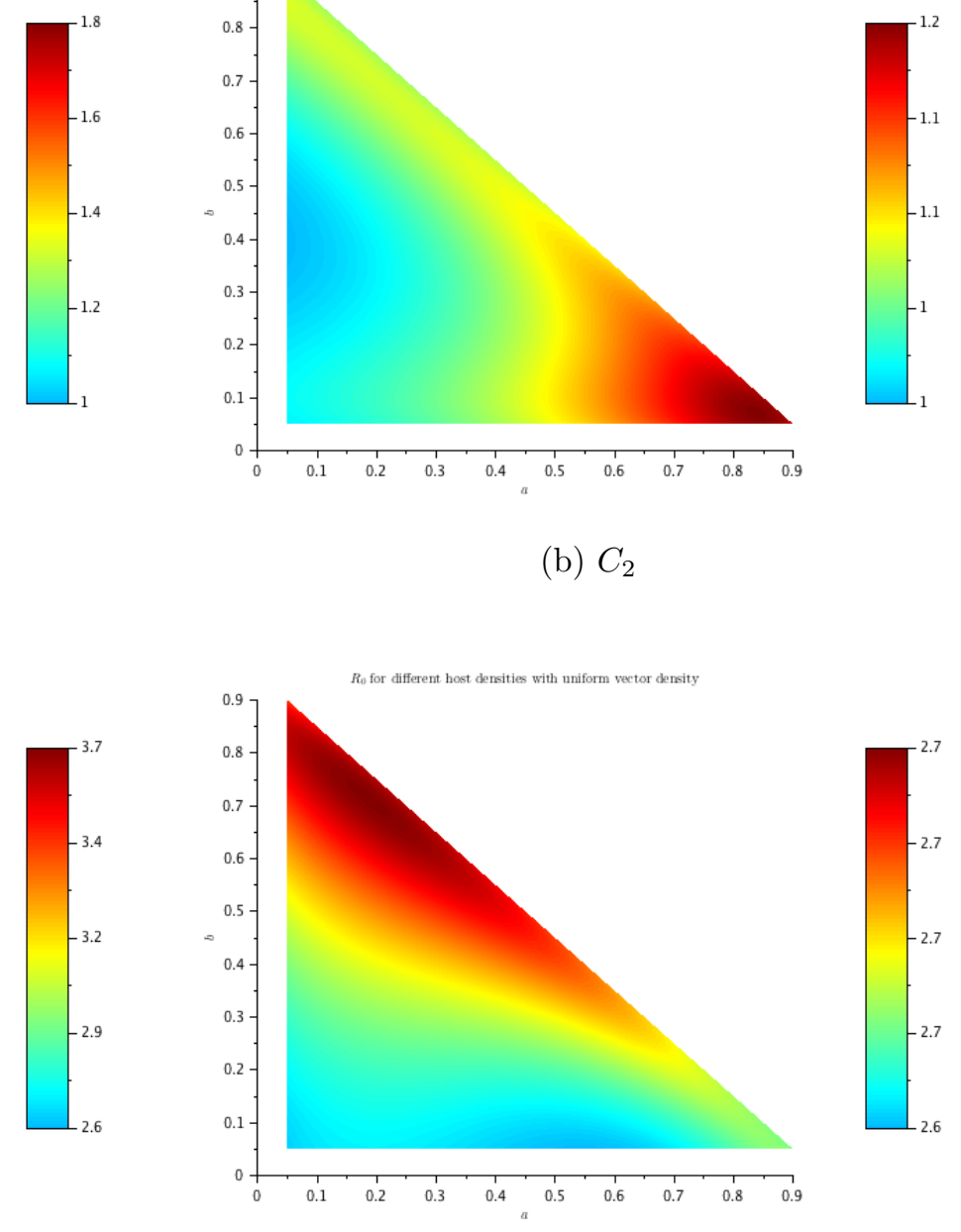

(d) $C_{2}$

Figure 1: Heat map of values $R_{0}$ for different host densities, given a uniform vector density. Figures (a) and (b) have $\hat{B}=\mathbb{1} \otimes \mathbb{1}$. For Figures (c) and (d) we took $\hat{B}=\mathbb{1} \otimes\left(\begin{array}{lll}4 & 1 & 2\end{array}\right)^{T}$. The $x$ axis denotes the fraction of hosts in the City Centre, while the $y$ axis denotes the fraction of hosts in Region 1. Notice also that in Figures (a) and (b), the dynamics given by $C_{1}$ is more sensitive than the one given by $C_{2}$ to host distribution inhomogeneities, and that it leads to the largest amplification of $R_{0}$ for very skewed host distributions. On the other hand, for uniform hosts distributions $C_{1}$ causes essentially no amplification, while the amplification implied by $C_{2}$ is more noticeable. For Figures (c) and (d), $C_{1}$ continues to yield the largest amplification, but now this is more noticeable only for very skewed host distributions. On the other hand, the amplifications yielded by $C_{2}$ continues to less dependent on the host distribution. 


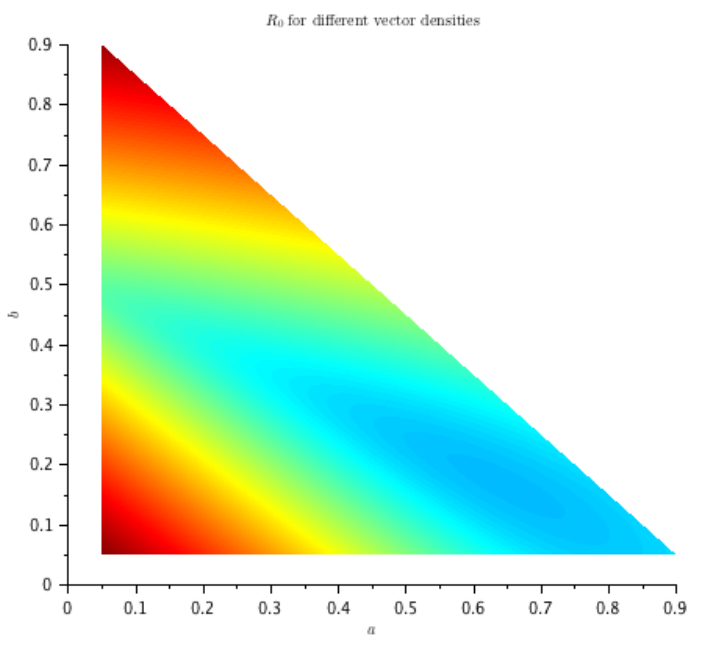

(a) $C_{1}$

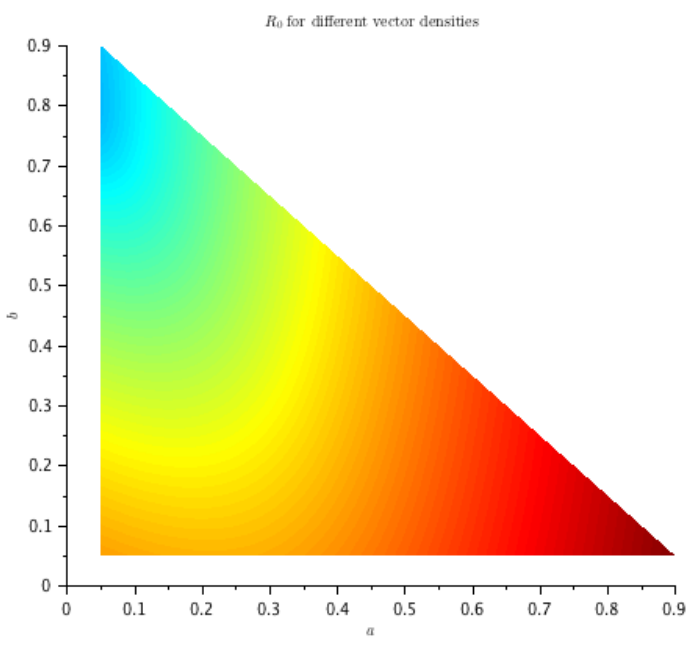

(c) $C_{1}$
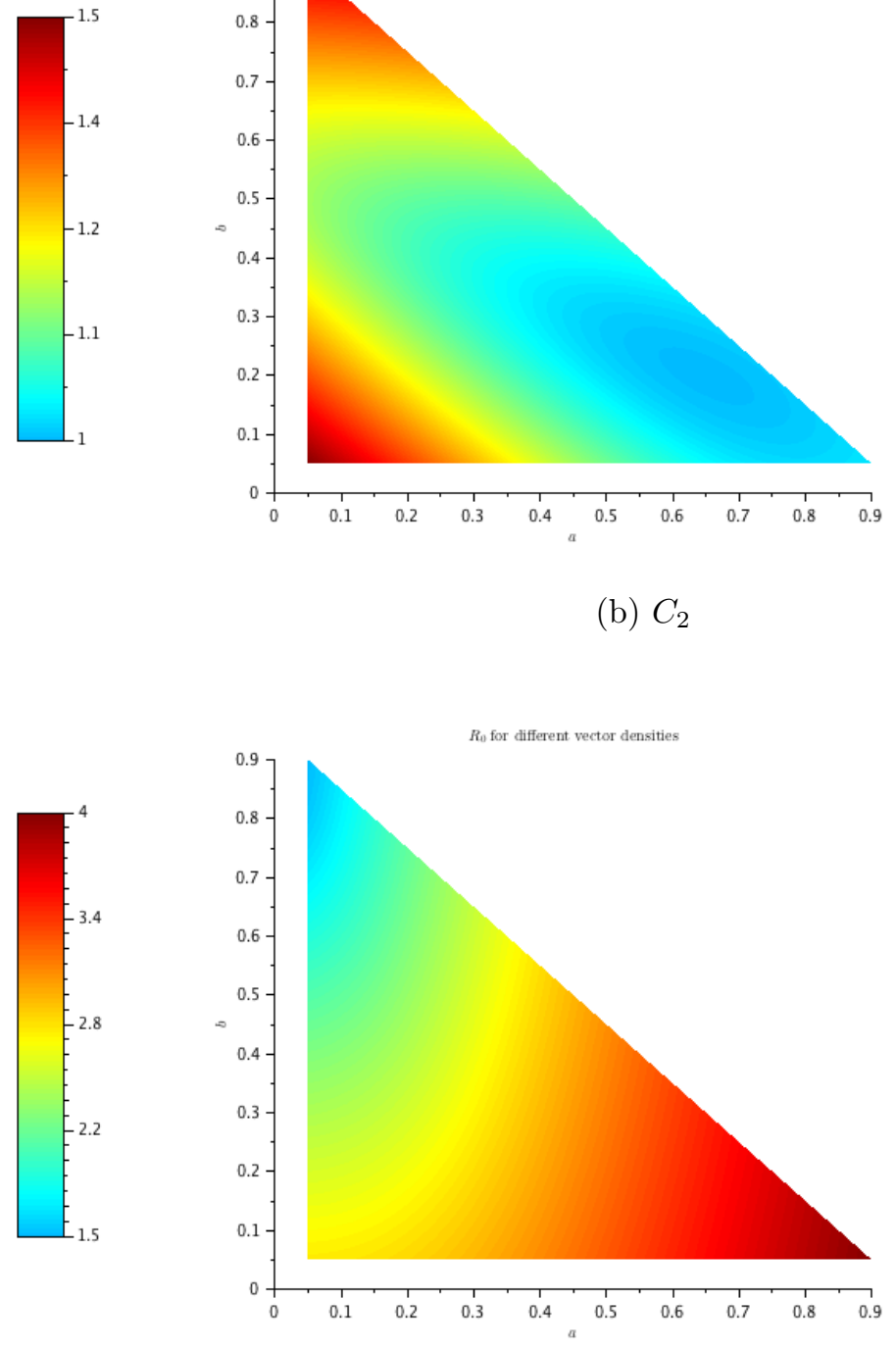

(d) $C_{2}$

Figure 2: Heat map of values $R_{0}$ for different vector densities, given an uniform host density. As in Figure 1, Figures (a) and (b) have $\hat{B}=\mathbb{1} \otimes \mathbb{1}$, and $C$ as indicated. For Figures (c) and (d), we again took $\hat{B}=\mathbb{1} \otimes\left(\begin{array}{lll}4 & 1 & 2\end{array}\right)^{T}$. Similarly, the $x$ axis denotes the fraction of vectors in the City Centre, while the $y$ axis denotes the fraction of vectors in Region 1. As in Figure 1, we have that $R_{0} \geq 1$ in Figures (a) and (b). Notice also that while $C_{1}$ continues to yield the largest $R_{0}$ amplification, the dependence on the host distribution is now more evenly spread. Also $C_{2}$ is now more sensitive to these variations, even if still leads to lower $R_{0}$ amplification ratios. 
Proposition 4.1. For an anomalous configuration, there is an open interval I such that $\frac{\bar{V}}{\bar{N}} \in I$, we have

$$
\max _{i} R_{0}^{\mathrm{loc}, i}<1<R_{0} .
$$

On the other hand, in an non-anomalous configuration where the maximum local $R_{0}$ is above unity, there are an open interval I such that

$$
R_{0}<1<\max _{i} R_{0}^{\mathrm{loc}, i}
$$

Remark 4.2. The combination of Proposition 4.1 with Proposition 3.2 together with the statistics of the computations performed yields an interesting picture:

1. In an anomalous configuration, a sufficient strong curfew will reduce $R_{0}$ below unity;

2. For a non-anomolous configuration with a maximal local $R_{0}$ above unity, but the global $R_{0}$ below unity, a sufficient strong curfew will increase $R_{0}$ above unity, thus worsening the situation.

Examples of these possibilities are given in Figure 3(a). However, notice that the behaviour of $R_{0}$ as a function of the curfew strength can be highly nonlinear, as indicated in the example in Figure 3(b).

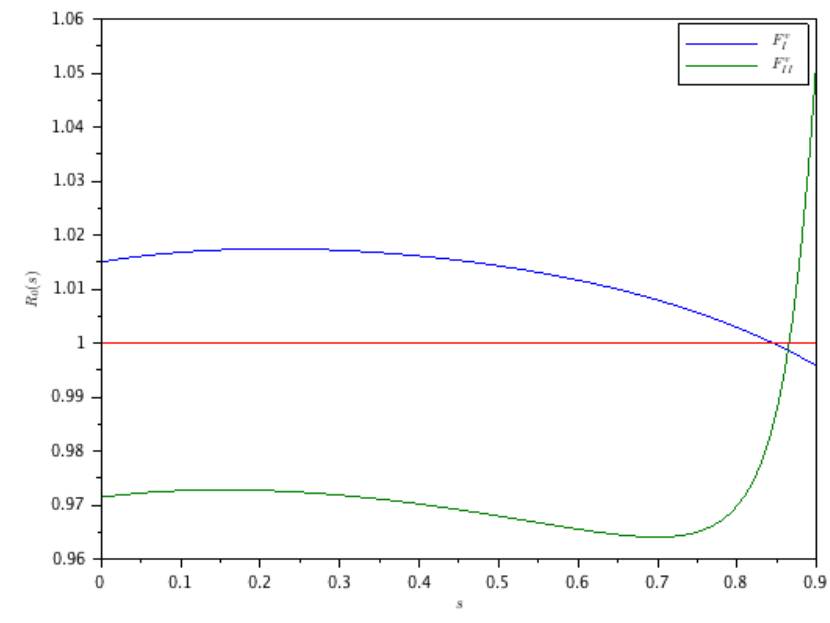

(a)

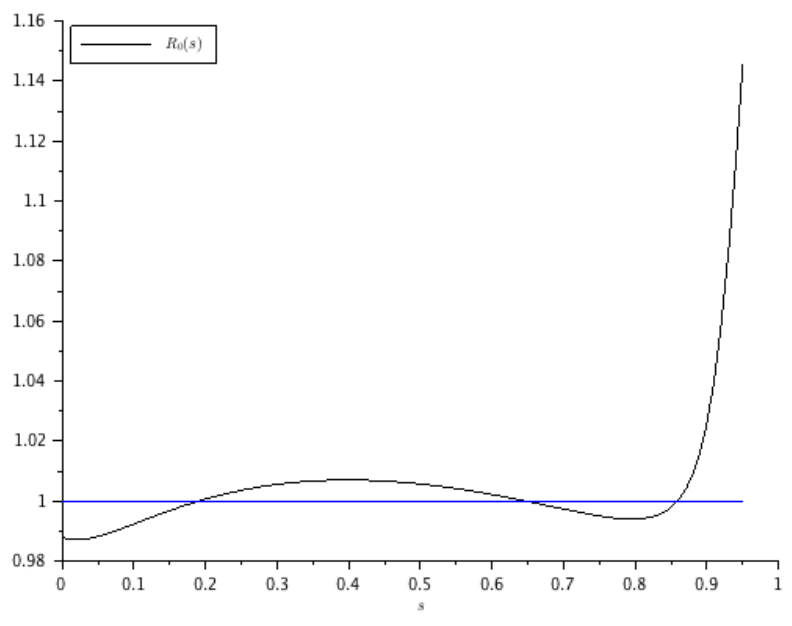

(b)

Figure 3: (a) Behaviour of $R_{0}$ for two different vector densities as a function of curfew strength, i.e., by considering the circulation matrix $\mathcal{C}_{s}=(1-s) C+s I$. (b) Similarly, but with the vector density given by $\hat{F}^{v}=(0.20 .30 .5)^{\dagger}$. The minimum $R_{0}^{\text {loc }}$ is 0.906 , and maximum is 1.384 . 


\subsubsection{Control}

We now present a brief study on the effect of vector population reduction on the value of $R_{0}$. For a given elimination effort $0 \leq \alpha \leq 1$, we consider three different policies: the first and simplest one is an uniform vector slay - the elimination effort is uniformly applied over the different regions; the second policy is a proportional one, where the effort is applied according to the infestation levels - this is equivalent to reduce $\widetilde{R_{0}}$ by $(1-\alpha)^{1 / 2}$; a region oriented one, where the elimination effort targets first a certain region, and if the infestation in this region is eliminated, the remain effort is spread uniformly in the other regions. The results of application of these three control policies are shown in Figure 4.

\subsection{Simulation with data from Rio de Janeiro}

We modelled the dynamics of a new arbovirus introduced in the city of Rio de Janeiro, Brazil, though System (10). Population data is available from the national census, while mosquito infestation data was obtained in two steps: we first obtained the relative infestation from a periodical report issued by the Brazilian Ministry of Health named Liraa (Levantamento de Índice Rápido de Infestação por Aedes) - Aedes Infestation Fast Index. We then have to select a host-vector ratio in order to have a fully determined vector population. Finally, the circulation matrix $C$ was obtained from data available from the transport authority of Rio .

The great metropolitan area of Rio was aggregated in 8 macro-regions: Centro, Sul, Norte, Oeste, Baixada, Niterói, Magé, Itaguaí-Centre, South,North,West are regions within the City of Rio, while the other comprise the great Metropolitan area of Rio de Janeiro, with most of people living in these regions working in the City of Rio. We considered a $20 \%$ host-vector ratio for this simulation, and used the biological parameters from Nishiura [2006]. The parameters corresponding to such aggregation can be found in B. In this framework, we found

$$
\widetilde{R_{0}}=0.875 \text { and } \quad R_{0}=2.566
$$

The local $R_{0}$ are given in Table 3 .

\begin{tabular}{l||c|c|c|c|c|c|c|c} 
Region & Centro & Sul & Norte & Oeste & Baixada & Niterói & Magé & Itaguaí \\
\hline Local $R_{0}$ & 0.948 & 0.760 & 0.681 & 0.487 & 0.616 & 1.149 & 2.618 & 1.991
\end{tabular}

Table 3: Local $R_{0}$ for the eight macro-regions considered in the simulation. Notice that the configuration is non-anomalous.

For the dynamical simulation, the initial condition was a single infected human in the Niterói region-for yet unaccounted reasons, almost all new infections arriving in Rio has the zeroth patient in this region.

The aggregated data for susceptible and infected hosts is shown in Figure 5. The results broken by region are presented in Figures 6 and 7. Notice that, although the initial infected patient arrives in Niterói so to speak, this region only see an outbreak roughly eight months after its arrival. The simulations also shows the delay in the outbreaks for the different regions

The final population distribution in terms of susceptible and recovered is shown in Figure 7. Notice that in two years we have that about ten percent of the population have became ill at some point. Notice also that the outbreak is confined to the Regions of Niterói, Magé, Itaguaíand 


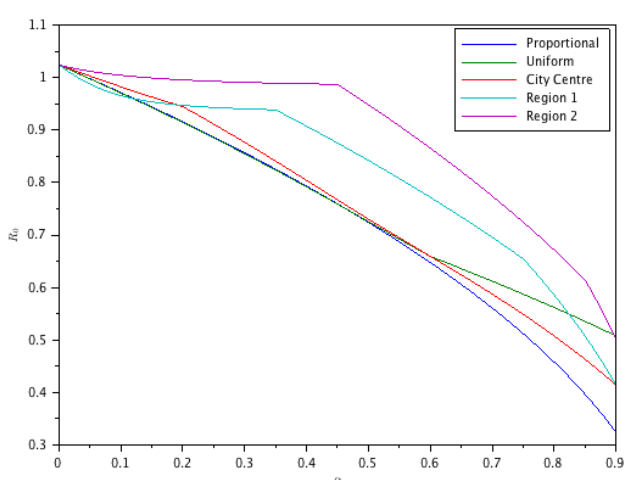

(a)

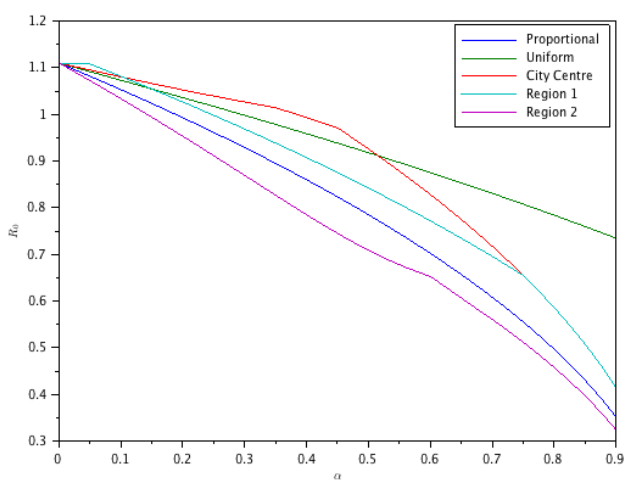

(c)

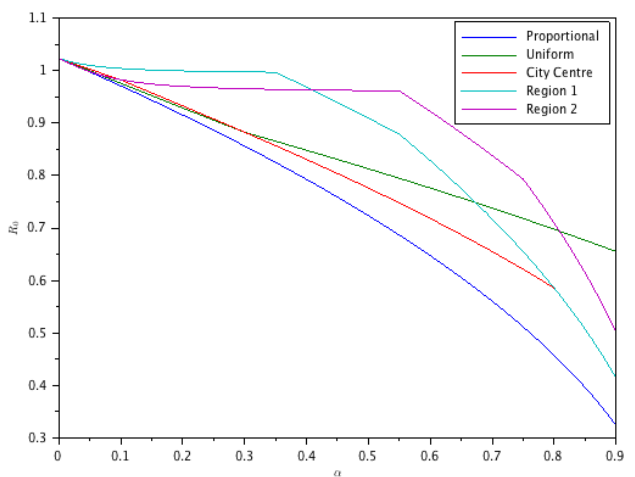

(e)

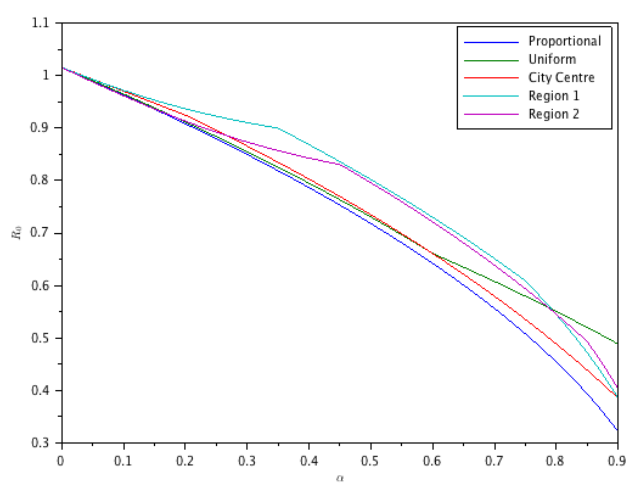

(b)

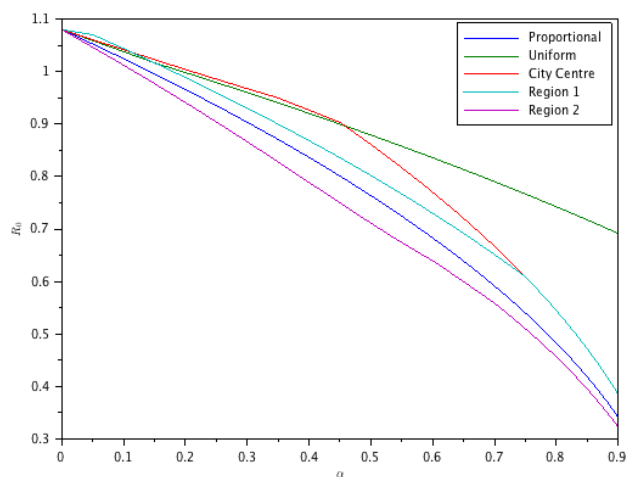

(d)

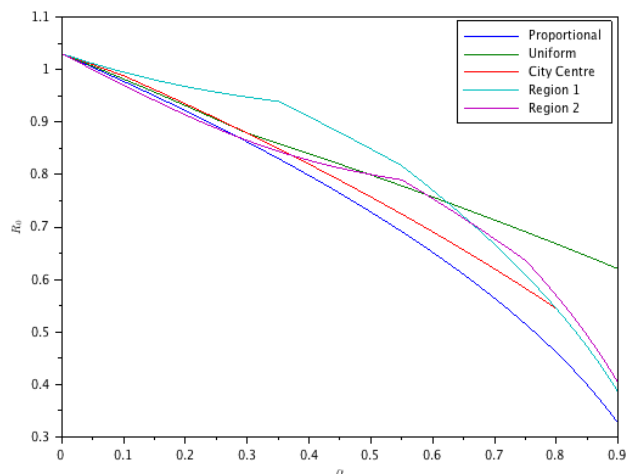

(f)

Figure 4: Control for the three different policies. The circulations matrices are $C_{1}$ in the first column, and $C_{2}$ in the second one. The vector densities are constant through lines. First line is (0.2 0.35 0.45); second line is (0.35 0.05 0.6); third line is the same as human density: (0.1 0.35 0.55). All other parameters as in Nishiura [2006]. Notice that (A) we have already a counterintuitive result: it is better to perform control in the rich area in this case. For (B) any control should work while as for (C), (D) and (F) control in the poorer region is more effective. Finally, in $(\mathrm{E})$ proportional control is more efficient. 


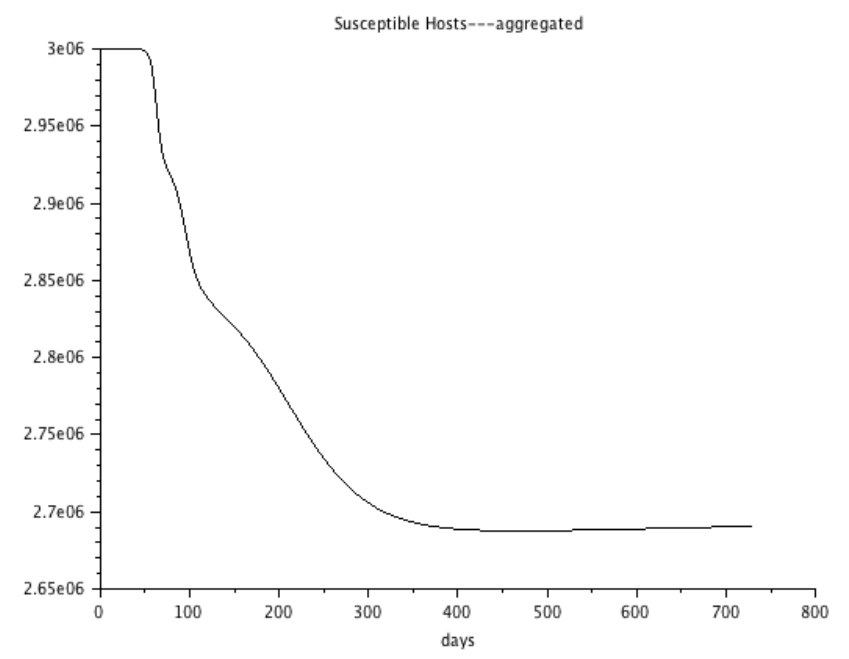

(a) Susceptible hosts

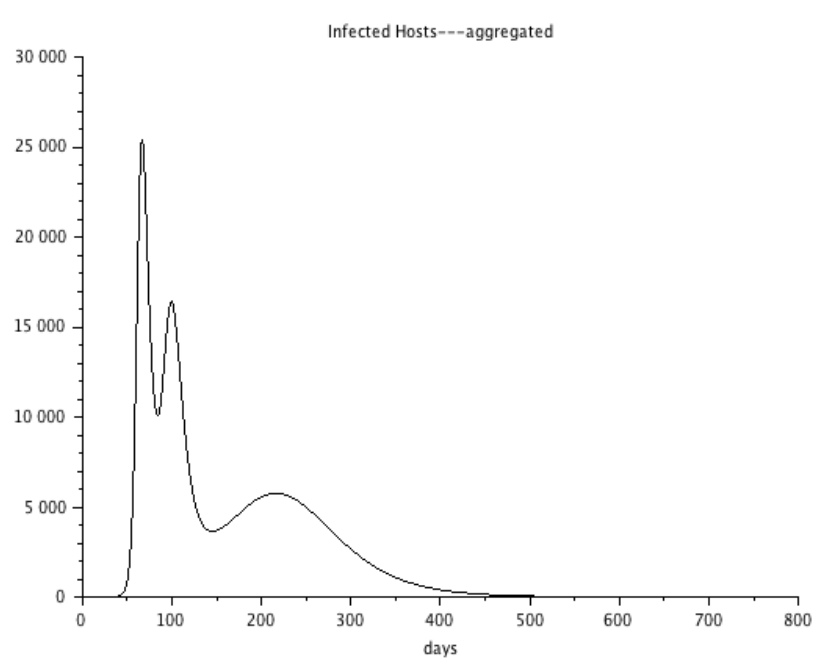

(b) Infected hosts

Figure 5: Aggregated data for susceptible and infected hosts.

Baixada. Notice also that while the first three are geographically contiguous, Baixada is quite far-around $60 \mathrm{~km}$ from Niterói, and even further from the other regions. Also, three other regions that are geographically close - the Centre and the South districts barely notice the outbreak.

\section{Conclusions}

In this work, we have presented a careful multi-scaling derivation of a multi-group based on the key assumptions that the hosts commute on a fast time-scale, whereas the vectors stay put. This derivation shows explicitly how the circulation patterns enter into the multi-group structure . The derivation is also based on an assumption of strong ergodicity of the migration urban dynamics. While this choice allows for the derivation of the simplest model, it should be verified against real data from urban dynamics.

For the computation of $R_{0}$, we assumed also that the heterogeneities arrising in the infectivity parameters are the same for both host-vector and vector-host transmission - a possible justification for this particular parametrisation arises when these differences are related to biting behaviour. In this framework, we introduce an auxiliary $n \times n$ matrix $L$ which encodes all the information for the model, and we obtain the result that $R_{0}=\widetilde{R_{0}} \sigma_{1}(L)$, where $\widetilde{R_{0}}$ is the classical basic reproductive number for a single patch. This suggests that $\sigma_{1}(L)$ might be seen as a heterogeneous correction to $\widetilde{R_{0}}$. Additionally, the matrix $L$ is written as a product of matrices that represent the different set of heterogeneities, and one can then consider different modelling scopes: full detail, biologically homogeneous $(\mathrm{BH})$, epidemiologically and biologically homogeneous (EBH), and Circulation Only $(\mathrm{CO})$. 


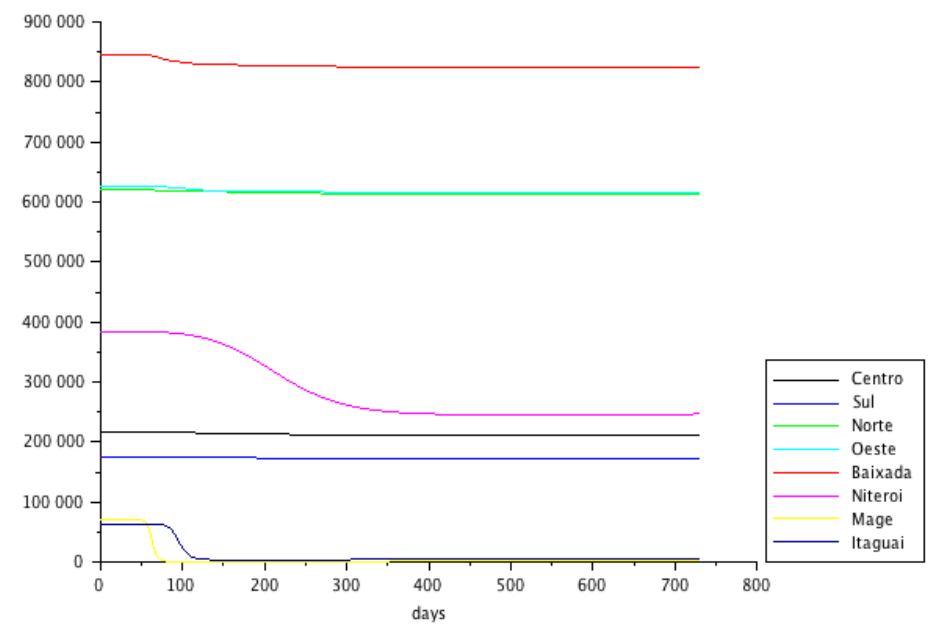

(a) Susceptible hosts

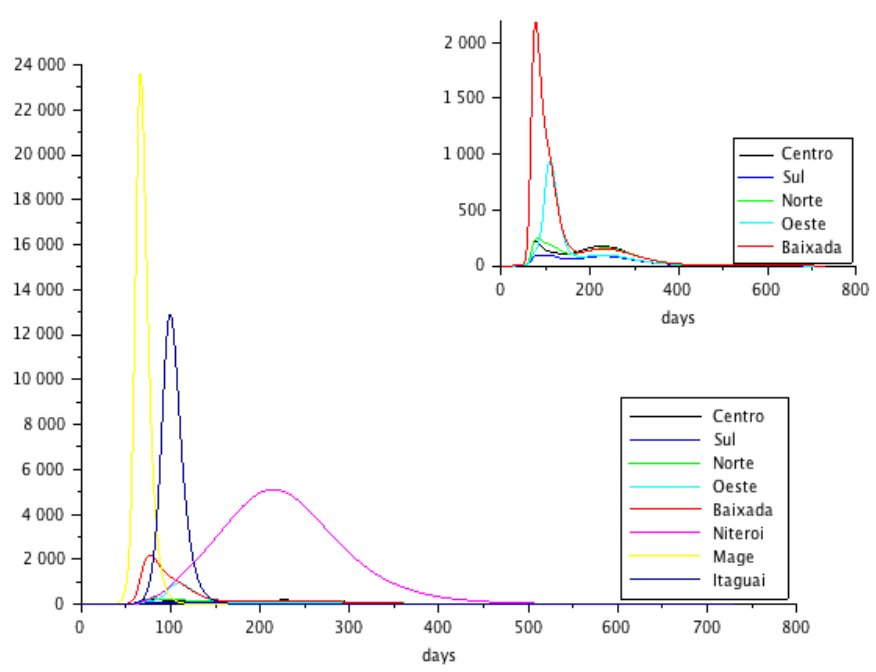

(b) Infected hosts

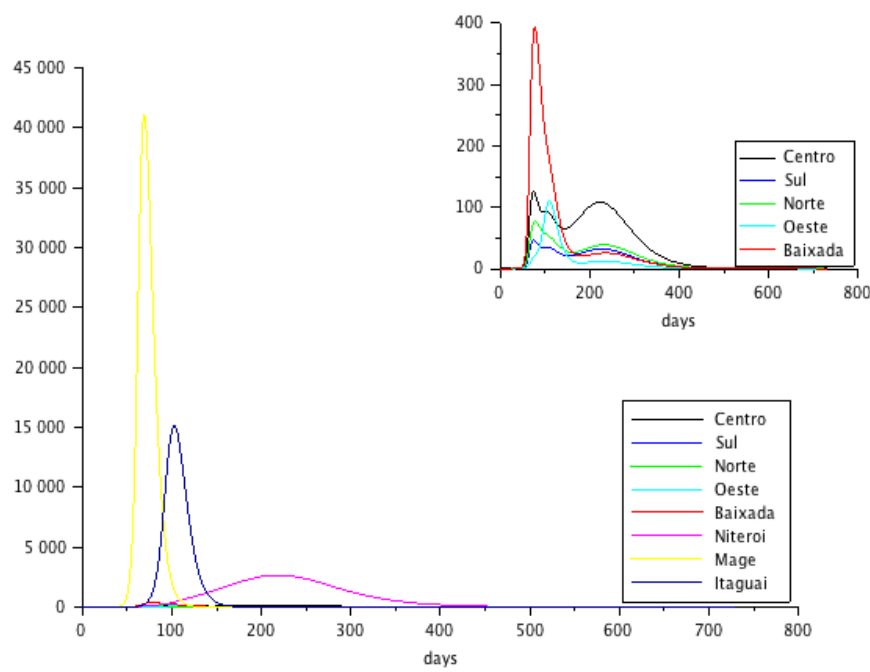

(c) Infected vectors

Figure 6: Data for each of the eight regions. For the infected populations, the inset shows the smallest populations outbreaks - this corresponds to the regions in the City, and the Metropolitan area of Baixada. 


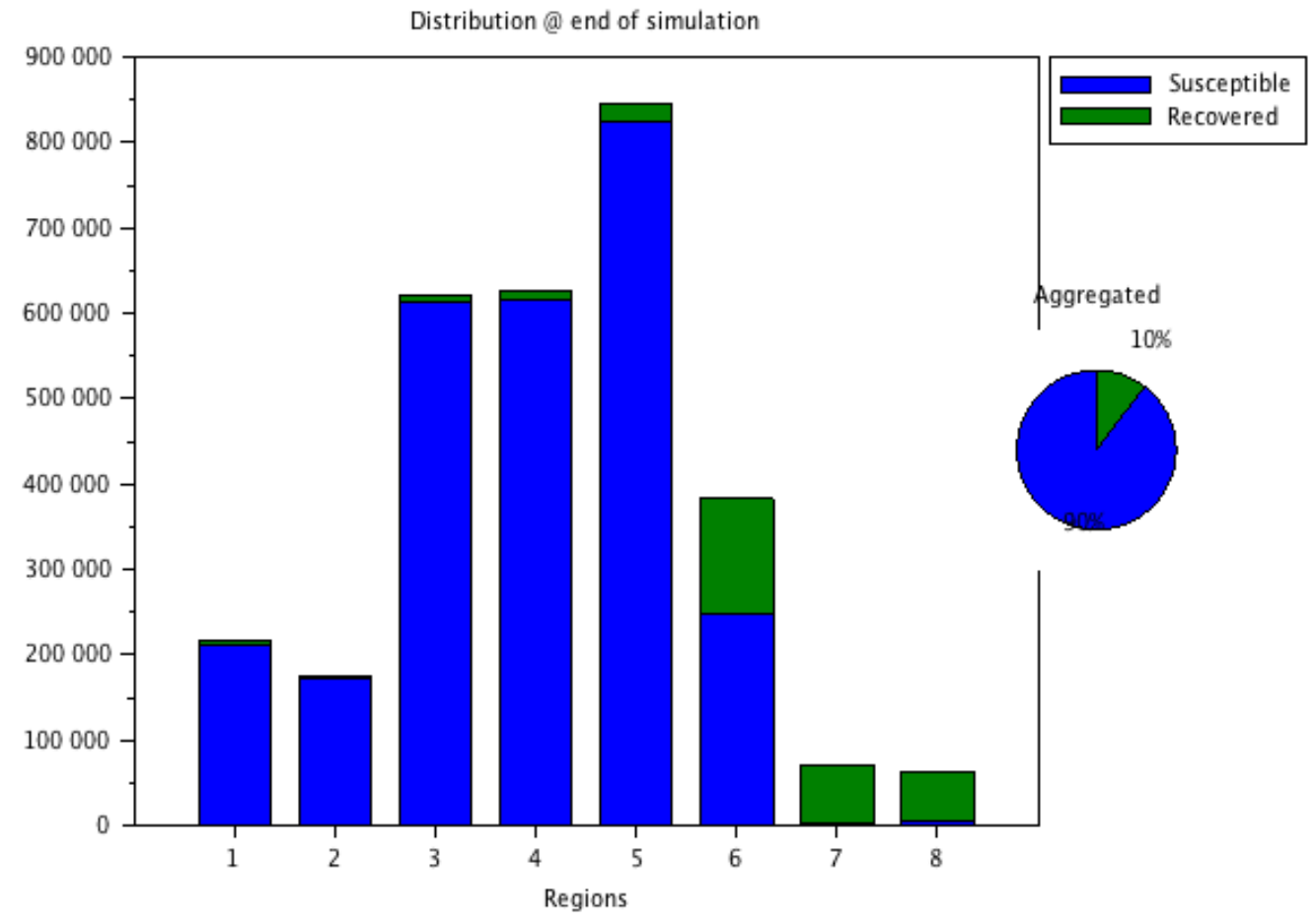

Figure 7: Epidemiological distribution at the end of the simulation. The number of infected is quite small, and hence only susceptible and recovered are accounted for. 
We have also shown that in the BH modelling scope, the "global" $R_{0}$ is always bounded below by $\widetilde{R_{0}}$. This might suggest that circulation is always worsening, but such a conclusion cannot be made in a clear cut way. Indeed, within the $\mathrm{BH}$ and $\mathrm{EBH}$ modelling scopes, we studied the dependence of $R_{0}$ with respect to density distribution of hosts and vectors. In these computations, some circulation patterns allowed to the existence of what we called anomalous configurations: configurations for which $R_{0}$ exceeds the maximal local $R_{0}$-i.e. the $R_{0}$ that one would obtain for each region should this region be disconnected from the others. This can be thought as curfew enforcement - which even in democratic societies can be encouraged through different measures. For these anomalous configuration, we show that there might exist infestation levels for which all local $R_{0}$ are below unity, yet $R_{0}$ is larger than unity. In this case, we showed that reducing circulation is beneficial and a strong enough curfew will eventually bring $R_{0}$ below one. On the other hand, we observed that in the majority of the configurations, the opposite is true: a sufficient reduction of circulation will increase $R_{0}$, and there might be cases where $R_{0}$ can be taken from below unity to above. We have also numerically studied different control strategies. As expected, a diverse range of heterogeneities patterns can lead to a corresponding diverse ordering of strategy efficiency. It should be also pointed out that there will situations where the lower bound given by Proposition 3.3 is not optimal. Further study of the model is needed in order to determine when this bound yields optima or quasi-optimal results.

Finally, we have also presented a simulation with data from the city of Rio de Janeiro. On one hand, this shows that the model can be effectively calibrated to real data, and that it has a richer transient dynamics than the single patch models. Such an enriched dynamics might be more compatible with the real data. On the other hand, much remains to be done in understanding this transient dynamics..

\section{A Proof of Proposition 3.1}

The last three equations of System (11) are

$$
\left\{\begin{array}{l}
\dot{S}^{h}=\operatorname{diag}\left(\mu^{h}\right)\left(F^{h}-S^{h}\right)-\operatorname{diag}\left(S^{h}\right)\left(B^{h} \circ C\right) \operatorname{diag}^{-1}\left(C^{T} N^{h}+A\right) I^{v} \\
\dot{I^{h}}=\operatorname{diag}\left(S^{h}\right)\left(B^{h} \circ C\right) \operatorname{diag}^{-1}\left(C^{T} N^{h}+A\right) I^{v}-\operatorname{diag}\left(\mu^{h}+\gamma\right) I^{h} \\
\dot{I^{v}}=\operatorname{diag}\left(N^{v}-I^{v}\right) \operatorname{diag}^{-1}\left(C^{T} N^{h}+A\right)\left(B^{v} \circ C\right)^{T} I^{h}-\operatorname{diag}\left(\mu^{v}\right) I^{v}
\end{array}\right.
$$

Recall that System $(20)$ has $\left(F_{h}, 0,0\right)$ as an equilibrium - the disease free equilibrium (DFE).

Since we are considering the case where $\hat{\zeta}=\mathbb{1}$, and $\hat{B}=\mathbb{1} \otimes \mathbb{1}, \hat{\omega}=\mathbb{1}, \hat{\mu}^{v}=\mathbb{1}$, the matrix $L$ is given by

$$
L=\operatorname{diag}\left(\hat{F}^{h}\right)^{1 / 2} C \operatorname{diag}\left(\hat{F}^{v}\right)^{1 / 2},
$$

and the Jacobian of System (20) at the DFE can be written

$$
J=\left(\begin{array}{ccc}
-\mu_{h} \operatorname{diag}(\mathbb{1}) & 0 & -\delta \operatorname{diag}\left(\hat{F}^{h}\right) C \\
0 & -\left(\mu_{h}+\gamma_{h}\right) \operatorname{diag}(\mathbb{1}) & \delta \operatorname{diag}\left(\hat{F}^{h}\right) C \\
0 & \alpha \operatorname{diag}\left(\hat{F}^{v}\right) C^{T} & -\mu_{v} \operatorname{diag}(\mathbb{1})
\end{array}\right)
$$

with $\delta=\frac{\bar{N}}{n}\left(\frac{\bar{N}+\bar{a}}{n}\right)^{-1} b \beta_{h}=\frac{\bar{N}}{\bar{N}+\bar{a}} b \beta_{h}$ and $\alpha=\frac{\bar{V}}{\bar{N}+\bar{a}} b \beta_{v}$. 
Thus the spectrum of $J$ is

$$
\sigma(J)=\left\{-\mu_{h}\right\} \cup \sigma(\hat{J}), \quad \hat{J}=\left(\begin{array}{cc}
-\left(\mu_{h}+\gamma_{h}\right) \operatorname{diag}(\mathbb{1}) & \delta \operatorname{diag}\left(\hat{F}^{h}\right) C \\
\alpha \operatorname{diag}\left(\hat{F}^{v}\right) C^{T} & -\mu_{v} \operatorname{diag}(\mathbb{1})
\end{array}\right) .
$$

The eigenvectors of the $2 n \times 2 n$ matrix $\hat{J}$ have a very special structure that can used to characterise local stability in terms of the uniform replication rate and the correction factor. Instrumental for the proof will be the balanced interaction matrix L. Its core is the elementary observation that $\hat{J}$ is similar to a Metzler matrix of the form

$$
\left(\begin{array}{cc}
-2 p \operatorname{diag}(\mathbb{1}) & q L \\
r L^{T} & -2 s \operatorname{diag}(\mathbb{1})
\end{array}\right) .
$$

Indeed, we have

$$
Q^{-1} \hat{J} Q=\left(\begin{array}{cc}
-\left(\mu_{h}+\gamma_{h}\right) \operatorname{diag}(\mathbb{1}) & \delta L \\
\alpha L^{T} & -\mu_{v} \operatorname{diag}(\mathbb{1})
\end{array}\right) \text { with } Q=\left(\begin{array}{cc}
\operatorname{diag}\left(\hat{F}^{h}\right)^{1 / 2} & 0 \\
0 & \operatorname{diag}\left(\hat{F}^{v}\right)^{1 / 2}
\end{array}\right) .
$$

Let $W$ a right singular vector of $L$ associated to a nonzero singular value $\theta$,

$$
L^{T} L W=\theta^{2} W .
$$

It is clear that $Z=L W$ is a left singular vector, also nonzero,

$$
L L^{T} Z=\theta^{2} Z \text {. }
$$

Let us try the following Anstaz in the spectral equation:

$$
\left(\begin{array}{cc}
-2 p \operatorname{diag}(\mathbb{1}) & q L \\
r L^{T} & -2 s, \operatorname{diag}(\mathbb{1})
\end{array}\right)\left(\begin{array}{c}
\rho L W \\
W
\end{array}\right)=\lambda\left(\begin{array}{c}
\rho L W \\
W
\end{array}\right)
$$

which gives

$$
-2 p \rho L W+q L W=\lambda \rho L W, \quad r \rho L^{T} L W-2 s W=\lambda W
$$

and hence two scalar equations

$$
-p \rho+q=\lambda \rho, \quad \theta^{2} r \rho-s=\lambda .
$$

Eliminating $\lambda$ we get a quadratic equation for $\rho$,

$$
\theta^{2} r \rho^{2}-2(s-p) \rho-q=0
$$

hence

$$
\theta^{2} r \rho_{ \pm}=s-p \pm \sqrt{(s-p)^{2}+\theta^{2} r q} .
$$

and

$$
\lambda_{ \pm}=-(p+s) \pm \sqrt{(s-p)^{2}+\theta^{2} r q} .
$$

Clearly the eigenvalues are real. The ones that correspond to the plus sign increase with $\theta$. The biggest eigenvalue is

$$
\lambda_{\max }=-(p+s)+\sqrt{(s-p)^{2}+\hat{\theta}^{2} r q}
$$


where

$$
\hat{\theta}=\sigma_{1}(L)
$$

is the highest singular value of $L$. We rewrite the expression inside the square root as

$$
(s-p)^{2}+\hat{\theta}^{2} r q=(p+s)^{2}+\hat{\theta}^{2} r q-4 p s .
$$

Therefore,

$$
\lambda_{\max }=-(p+s)+\sqrt{(p+s)^{2}+\hat{\theta}^{2} r q-4 p s}
$$

$\lambda_{\max }$ turns from negative to positive when the expression $\hat{\theta}^{2} r q-4 p s$ changes sign.

Thanks to relation (15), we have $\hat{\theta}=\frac{R_{0}}{\widetilde{R}_{0}}$. Thus $\hat{\theta}^{2} r q-4 p s=4 p s\left(\hat{\theta}^{2} \frac{r q}{4 p s}-1\right)=4 p s\left(\frac{R_{0}^{2}}{\widetilde{R}_{0}^{2}} \frac{r q}{4 p s}-1\right)$

Finally, substituting into $r q / 4 p s$ the values

$$
r=\alpha, q=\delta, 2 p=\mu_{h}+\gamma_{h}, 2 s=\mu_{v}
$$

a "miracle" happens:

$$
\frac{r q}{4 p s}=\frac{\alpha \delta}{\mu_{v}\left(\mu_{h}+\gamma_{h}\right)}=\widetilde{R}_{0}^{2}
$$

The expression (17) for the biggest eigenvalue of $J$ follows from inserting (16) in (30).

\section{B Data from Rio de Janeiro}

\section{Human population}

\begin{tabular}{|l|c|c|c|c|c|c|c|c|}
\hline Region & Centro & Sul & Norte & Oeste & Baixada & Niterói & Magé & Itaguaí \\
\hline Population & 792486 & 640051 & 2265444 & 2285822 & 3088011 & 1400785 & 260854 & 233180 \\
\hline
\end{tabular}

\section{Vector population}

\begin{tabular}{|l|c|c|c|c|c|c|c|c|}
\hline Region \# & Centro & Sul & Norte & Oeste & Baixada & Niterói & Magé & Itaguaí \\
\hline Infestation & 0.928 & 0.48 & 1.36 & 0.7 & 1.52 & 2.4 & 2.32 & 1.2 \\
\hline
\end{tabular}

\section{Circulaton Matrix}

$$
C=\left(\begin{array}{cccccccc}
0.732125236 & 0.070458027 & 0.111726895 & 0.050154829 & 0.019869121 & 0.015296169 & 0.000369723 & 0 \\
0.222597887 & 0.712299489 & 0.023501252 & 0.035295625 & 0.00320287 & 0.003029446 & 7.34316 E-05 \\
0.083882894 & 0.018216738 & 0.869539481 & 0.014608174 & 0.010300409 & 0.003318996 & 7.72476 E-05 & 5.60597 E-05 \\
0.065137618 & 0.028101488 & 0.021037509 & 0.87320185 & 0.007269157 & 0.003050106 & 4.94352 E-05 & 0.002152836 \\
0.07135564 & 0.020688398 & 0.033865164 & 0.017163799 & 0.851849621 & 0.002864951 & 0.0013805 & 0.000831927 \\
0.064548093 & 0.013054109 & 0.006784767 & 0.002372241 & 0.001390649 & 0.911082 & 0 & 0.000768141 \\
0.016484317 & 0.006248706 & 0.008568011 & 0.002273302 & 0.035958812 & 0.003427204 & 0.927039647 & 0 \\
0.008135346 & 0.001938417 & 0.002101381 & 0.023561197 & 0.005403551 & 0 & 0 & 0.958860108
\end{array}\right)
$$




\section{References}

Adams, B., Kapan, D. D., 2009. Man bites mosquito: understanding the contribution of human movement to vector-borne disease dynamics. PLoS One 4 (8), e6763.

Adler, F. R., 9 1992. The effects of averaging on the basic reproduction ratio. Mathematical Biosciences 111 (1), 89-98.

Alvim, M., Iggidr, A., Koiler, J., Sallet, G., Penna, M. L. F., Souza, M. O., 2013. Onset of a vector borne disease due to human circulation - uniform, local and network reproduction ratios. Preprint HAL: https://hal.inria.fr/hal-00839351v2.

Andreasen, V., Christiansen, F. B., Oct 1989. Persistence of an infectious disease in a subdivided population. Mathematical Biosciences 96 (2), 239-253.

Añez, G., Rios, M., 2013. Dengue in the united states of america: a worsening scenario? BioMed research international 2013.

Arino, J., 2009. Diseases in metapopulations. In: Ma, Z., Zhou, Y., Wu, J. (Eds.), Modeling and Dynamics of Infectious Diseases. Vol. 11 of Series in Contemporary Applied Mathematics. World Scientific, pp. 65-123, also CDM Preprint Series report 2008-04.

Arino, J., Ducrot, A., Zongo, P., 2012. A metapopulation model for malaria with transmissionblocking partial immunity in hosts. Journal of mathematical biology 64 (3), 423-448.

Arino, J., van den Driessche, P., 2003. A multi-city epidemic model. Math. Popul. Stud. 10 (3), $175-193$.

Bailey, N., 1975. The Mathematical Theory of Infectious Diseases and its Applications. Griffin,London.

Berman, A., Plemmons, R. J., 1979. Nonnegative matrices. The Mathematical Sciences, Classics in Applied Mathematics 9.

Bhatt, S., Gething, P. W., Brady, O. J., Messina, J. P., Farlow, A. W., Moyes, C. L., Drake, J. M., Brownstein, J. S., Hoen, A. G., Sankoh, O., et al., 2013. The global distribution and burden of dengue. Nature 496 (7446), 504-507.

Bichara, D., Kang, Y., Castillo-Chavez, C., Horan, R., Perrings, C., 2015. Sis and sir epidemic models under virtual dispersal. Bulletin of mathematical biology 77 (11), 2004-2034.

Bowong, S., Dumont, Y., Tewa, J. J., 2013. A patchy model for chikungunya-like diseases. Biomath 2 (1), 1307237.

CDC, 2016. Where has chikungunya virus been found? https://www.cdc.gov/chikungunya/ geo/, accessed on 7th November 2016.

Cosner, C., Beier, J., Cantrell, R., Impoinvil, D., Kapitanski, L., Potts, M., Troyo, A., Ruan, S., 2009. The effects of human movement on the persistence of vector-borne diseases. Journal of Theoretical Biology 258 (4), 550-560. 
Diekmann, O., Heesterbeek, J., Metz, J., 1990. On the definition and the computation of the basic reproduction ratio $R_{0}$ in models for infectious diseases in heterogeneous populations. J. Math. Biol. 28 (4), 365-382.

Dietz, K., 1975. Transmission and control of arbovirus diseases. In: Ludwig, D., Cooke, K. L. (Eds.), Epidemiology. SIAM, pp. 104-121.

Dushoff, J., Levin, S., 1995. The effects of population heterogeneity on disease invasion. Mathematical Biosciences 128 (1-2), 25-40.

Esteva, L., Vargas, C., 1998. Analysis of a dengue disease transmission model. Math. Biosci. $150(2), 131-151$.

Eubank, S., Guclu, H., Kumar, V. A., Marathe, M. V., Srinivasan, A., Toroczkai, Z., Wang, N., 2004. Modelling disease outbreaks in realistic urban social networks. Nature 429 (6988), 180-184.

Gatto, M., Mari, L., Bertuzzo, E., Casagrandi, R., Righetto, L., Rodriguez-Iturbe, I., Rinaldo, A., Nov 2012. Generalized reproduction numbers and the prediction of patterns in waterborne disease. Proc Natl Acad Sci U S A 109 (48), 19703-19708.

Gubler, D. J., Feb 2002. Epidemic dengue/dengue hemorrhagic fever as a public health, social and economic problem in the 21st century. Trends Microbiol 10 (2), 100-103.

Hasibeder, G., Dye, C., 2 1988. Population dynamics of mosquito-borne disease: Persistence in a completely heterogeneous environment. Theoretical Population Biology 33 (1), 31-53.

Hethcote, H. W., Yorke, J., 1984. Gonorrhea : transmission dynamics and control. Vol. 56 of Lect. Notes Biomath. Springer-Verlag.

Honorio, N. A., Silva, W. d. C., Leite, P. J., Goncalves, J. M., Lounibos, L. P., Lourenco-de Oliveira, R., Mar 2003. Dispersal of aedes aegypti and aedes albopictus (diptera: Culicidae) in an urban endemic dengue area in the state of rio de janeiro, brazil. Mem Inst Oswaldo Cruz 98 (2), 191-198.

Horn, R. A., Johnson, C. R., 1990. Matrix Analysis. CUP.

Horn, R. A., Johnson, C. R., 1991. Topics in Matrix Analysis. CUP.

Iggidr, A., Sallet, G., Souza, M. O., 2016. On the dynamics of a class of multi-group models for vector-borne diseases. Journal of Mathematical Analysis and Applications 441 (2), 723-743.

Kraemer, M. U., Sinka, M. E., Duda, K. A., Mylne, A. Q., Shearer, F. M., Barker, C. M., Moore, C. G., Carvalho, R. G., Coelho, G. E., Van Bortel, W., et al., 2015. The global distribution of the arbovirus vectors aedes aegypti and ae. albopictus. Elife 4, e08347.

Lajmanovich, A., Yorke, J., 1976. A deterministic model for gonorrhea in a nonhomogeneous population. Math. Biosci. 28, 221-236.

Lambrechts, L., Scott, T. W., Gubler, D. J., 2010. Consequences of the expanding global distribution of aedes albopictus for dengue virus transmission. PLoS Negl Trop Dis 4 (5), e646. 
Lawson, A. B., Leimich, P., 2000. Approaches to the space-time modelling of infectious disease behaviour. Mathematical Medicine and Biology 17 (1), 1-13.

Liang, G., Gao, X., Gould, E. A., 2015. Factors responsible for the emergence of arboviruses; strategies, challenges and limitations for their control. Emerging microbes \& infections 4 (3), e18.

Maciel-de Freitas, R., Lourenço-de Oliveira, R., 2009. Presumed unconstrained dispersal of aedes aegypti in the city of rio de janeiro, brazil. Revista de Saúde Pública 43 (1), 8-12.

Martens, W., Jetten, T., Rotmans, J., Niessen, L., 1995. Climate change and vector-borne diseases: a global modelling perspective. Global environmental change 5 (3), 195-209.

Nishiura, H., 2006. Mathematical and statistical analyses of the spread of dengue. Dengue Bulletin $30,51-67$.

Nold, A., 1980. Heterogeneity in disease-transmission modeling. Mathematical Biosciences 52 (3), $227-240$.

Norris, J. R., 1997. Markov Chains. Cambridge University Press, cambridge Books Online.

Padmanabha, H., Durham, D., Correa, F., Diuk-Wasser, M., Galvani, A., 08 2012. The interactive roles of aedes aegypti super-production and human density in dengue transmission. PLoS Negl Trop Dis 6 (8), e1799.

Pereira, R. H. M., Schwanen, T., 2015. Commute time in brazil (1992-2009): differences between metropolitan areas, by income levels and gender. Tech. rep., IPEA.

Rocha, F., Aguiar, M., Souza, M. O., Stollenwerk, N., 2013. Time-scale separation and center manifold analysis describing vector-borne disease dynamics. International Journal of Computer MathematicsForthcoming.

Samarasekera, U., Triunfol, M., 2016. Concern over zika virus grips the world. The Lancet 387 (10018), 521-524.

Shuai, Z., van den Driessche, P., 2013. Global stability of infectious disease models using Lyapunov functions. SIAM Journal on Applied Mathematics 73 (4), 1513-1532.

Smith, D. L., Dushoff, J., McKenzie, F. E., 2004. The risk of a mosquito-borne infection in a heterogeneous environment. PLoS Biol 2 (11), e368.

Souza, M. O., 2014. Multiscale analysis for a vector-borne epidemic model. Journal of Mathematical Biology 68 (5), 1269-1293.

Stoddard, S. T., Forshey, B. M., Morrison, A. C., Paz-Soldan, V. A., Vazquez-Prokopec, G. M., Astete, H., Reiner, R. C., Vilcarromero, S., Elder, J. P., Halsey, E. S., et al., 2013. House-tohouse human movement drives dengue virus transmission. Proceedings of the National Academy of Sciences 110 (3), 994-999.

Stoddard, S. T., Morrison, A. C., Vazquez-Prokopec, G. M., Paz Soldan, V., Kochel, T. J., Kitron, U., Elder, J. P., Scott, T. W., 07 2009. The role of human movement in the transmission of vector-borne pathogens. PLoS Negl Trop Dis 3 (7), e481 EP -. 
Teschl, G., 2012. Ordinary differential equations and dynamical systems. Vol. 140. American Mathematical Society Providence, RI.

Teurlai, M., Huy, R., Cazelles, B., Duboz, R., Baehr, C., Vong, S., 12 2012. Can human movements explain heterogeneous propagation of dengue fever in Cambodia? PLoS Negl Trop Dis 6 (12), e1957 EP -.

Thieme, H. R., 2003. Mathematics in population biology. Princeton Series in Theoretical and Computational Biology. Princeton University Press, Princeton, NJ.

van den Driessche, P., Watmough, J., 2002. reproduction numbers and sub-threshold endemic equilibria for compartmental models of disease transmission. Math. Biosci. 180, 29-48.

Xiao, Y., Zou, X., 2014. Transmission dynamics for vector-borne diseases in a patchy environment. Journal of Mathematical Biology 69 (1), 113-146. 